\title{
MEASURE-VALUED SOLUTIONS TO INITIAL-BOUNDARY VALUE PROBLEMS FOR CERTAIN SYSTEMS OF CONSERVATION LAWS: EXISTENCE AND DYNAMICS
}

\author{
HERMANO FRID
}

\begin{abstract}
A framework for studying initial-boundary value problems for systems of conservation laws, in what concerns to the existence of measure-valued solutions and their asymptotic behavior, is developed here with the helpful introduction of a class of flux maps which allow a rather complete treatment of these questions including systems of practical importance as those arising in multiphase flow in porous media. The systems of this class may, in general, admit umbilic points, submanifolds where genuine nonlinearity fails, as well as elliptic regions. We prove the existence of measure-valued solutions by using the vanishing viscosity method and, also, finite difference schemes. The main result about the dynamics of the measure-valued solutions is that for certain special boundary values, given by constant states, the time-averages of these $\mathrm{m}-\mathrm{v}$ solutions converge weakly to the Dirac measure concentrated at those states, for a.e. space variable. The rate of convergence of the time-averages of the expected values can be estimated by properties of the flux maps only.
\end{abstract}

\section{INTRODUCTION}

In this work we will be concerned with the development of a framework for studying initial-boundary value problems for systems of conservation laws with regard to the existence of measure-valued $(\mathrm{m}-\mathrm{v})$ solutions, whose definition in the context of Cauchy problems was first proposed by DiPerna [2], and their asymptotic behavior. To this purpose we introduce a special class of flux maps (the $K$-fluxes and $\Delta^{i_{1}, i_{2}}$-fluxes defined below) which allow a rather extensive treatment of the referred questions including systems modelling practical problems (cf. [3]). Although here we will always be dealing with systems possessing flux maps which belong to this special class, the techniques presented here for studying important aspects of these problems (e.g., the assumption of the boundary conditions, the asymptotic behavior, etc.) can be applied to a much larger set of systems. So, what we intend here, first of all, is to give a systematic presentation of these techniques applied to the concrete case of the $K$-fluxes and $\Delta^{i_{1}, i_{2}}$-fluxes (see Definitions 1.1, 1.3, below). The question of the existence is treated here in two different ways: the vanishing viscosity method and the approximation by finite difference schemes. The important steps in this study are the proof of the invariancy of the region of interest by the

Received by the editors March 17, 1994.

1991 Mathematics Subject Classification. Primary 35L60, 35L50, 35B40; Secondary 76T05.

Key words and phrases. Measure-valued solutions, systems of conservation laws, systems of mixed type, initial-boundary value problems.

Research partially supported by CNPq-Brazil, proc. 302307/86-9. 
approximation scheme, and the proof of the assumption of the boundary conditions in the required way (cf. Definition 1.6). As to the question of the asymptotic behavior, we carry out this study by means of the analysis of the time-averages of the $\mathrm{m}-\mathrm{v}$ solutions for each fixed space variable, which form a one-parameter family of probability measures. We reduce this problem to prove the weak convergence of a family of probability measures to a Dirac measure, once we know that the elements of this family applied to a certain function converge to a Dirac measure applied to that function. This study also gives estimates for the rates of convergence of the expected values of the dependent variables. It is interesting to observe that the same asymptotic behavior of the expected values of the $\mathrm{m}-\mathrm{v}$ solutions is already present in the approximate solutions obtained by the finite difference schemes used here. This allows the accomplishment of numerical experiments to verify the estimates for the rates of convergence and the obtainment of better ones; we do not carry out this investigation here.

This work is organized in the following way. In this section we will state the basic definitions and some important properties. In particular, we state the initialboundary value problem which we will be treating here. In section 2 we prove the existence of $\mathrm{m}-\mathrm{v}$ solutions to this problem, in the sense of Definition 1.5, below, by the vanishing viscosity method. In section 3 we prove the existence of $\mathrm{m}-\mathrm{v}$ solutions by two finite difference schemes: one discretizing both space and time variables, and the other discretizing only the space variable. Finally, in section 4 we make the study about the asymptotic behavior of these $\mathrm{m}-\mathrm{v}$ solutions.

Let $K=\prod_{i=1}^{n}[0,1]$ be the $n$-dimensional unit cube and $\mathbf{u}$ denote a generic element of $K$. Let $\mathbf{e}$ denote a vertex of $K$, that is, $\mathbf{e}=\left(\delta_{1}, \ldots, \delta_{n}\right)$ where $\delta_{i}=0$ or $1, i=1, \ldots, n$. For $\varepsilon_{0}>0$, set $U_{\varepsilon_{0}}=\prod_{i=1}^{n}\left[-\varepsilon_{0}, \varepsilon_{0}\right]$ and denote

$$
K\left(\mathbf{e} ; \varepsilon_{0}\right)=K \cap\left(\mathbf{e}+U_{\varepsilon_{0}}\right) .
$$

1.1 Definition. A $K$-flux of first kind is a continuous map $\mathbf{f}: K \rightarrow K$, $\mathbf{f}=$ $\left(f_{1}, \ldots, f_{n}\right)$ satisfying:

(i) $f_{i}\left(u_{1}, \ldots, u_{i-1}, \delta_{i}, u_{i+1}, \ldots, u_{n}\right)=\delta_{i}$, where $\delta_{i}=0$ or $1, i=1, \ldots, n$;

(ii) for a certain $\varepsilon_{0}>0$ and a certain vertex e of $K, \mathbf{f}$ is a homeomorphism of $K\left(\mathbf{e} ; \varepsilon_{0}\right)$ onto its image by $\mathbf{f}$.

The vertex e in (ii) of Definition 1.1 will be called a vertex of coerciveness for the $K$-flux $\mathbf{f}$. We say that the $K$-flux $\mathbf{f}$ is of class $C^{k}$ if $\mathbf{f}$ can be extended as a map $C^{k}$ to a neighborhood of $K$. We denote by $\mathcal{F}^{+}(K)$ the set of all $K$-fluxes of first kind.

Let us define $\mathcal{S}(K)$, a set of symmetries of $K$ given by

$$
\mathcal{S}(K)=\left\{S: K \rightarrow K \mid S_{i}(\mathbf{u})=u_{i} \text { or } 1-u_{i}\right\}
$$

The set $\mathcal{S}(K)$ possesses, of course, $2^{n}$ elements. For $S \in \mathcal{S}(K)$ we set

$$
S=\left[j_{1}, \ldots, j_{p}\right]
$$

if $\left\{j_{1}, \ldots, j_{p}\right\}$ is the set of indexes $i$ such that $S_{i}(u)=u_{i}$. 
1.2 Definition. We say that $\mathbf{g}: K \rightarrow K$ is a $K$-flux if there exist $S \in \mathcal{S}(K)$ and $\mathbf{f} \in \mathcal{F}^{+}(K)$ such that $\mathbf{g}=S \mathbf{f}$.

We denote by $\mathcal{F}(K)$ the set of $K$-fluxes.

Let us define the prisms $\Delta^{i_{1}, i_{2}}$, for $n \geq 2,1 \leq i_{1}<i_{2} \leq n$, by

$$
\Delta^{i_{1}, i_{2}}=\left\{\mathbf{u} \in K \mid \quad u_{i_{1}}+u_{i_{2}} \leq 1\right\} .
$$

We also denote

$$
\Delta^{i_{1}, i_{2}}\left(\mathbf{e} ; \varepsilon_{0}\right)=\Delta^{i_{1}, i_{2}} \cap\left(\mathbf{e}+U_{\varepsilon_{0}}\right),
$$

for $\varepsilon_{0}$ and a vertex $\mathbf{e}$ of $\Delta^{i_{1}, i_{2}}$.

1.3 Definition. A $\Delta^{i_{1}, i_{2}}$-flux of first kind is a map $\mathbf{f}: \Delta^{i_{1}, i_{2}} \rightarrow \Delta^{i_{1}, i_{2}}$ satisfying:

(i) $f_{i}\left(u_{1}, \ldots, u_{i-1}, \delta_{i}, u_{i+1}, \ldots, u_{n}\right)=\delta_{i}$, where $\delta_{i}=0$ or $1, i=1, \ldots, n$, and $\left(u_{1}, \ldots, u_{i-1}, \delta_{i}, u_{i+1}, \ldots, u_{n}\right) \in \Delta^{i_{1}, i_{2}}$

(ii) $f_{i_{1}}(\mathbf{u})+f_{i_{2}}(\mathbf{u})=1$, if $u_{i_{1}}+u_{i_{2}}=1$;

(iii) for a certain $\varepsilon_{0}$ and a vertex $\mathbf{e}$ of $\Delta^{i_{1}, i_{2}}, \mathbf{f}$ is a homeomorphism of $\Delta^{i_{1}, i_{2}}\left(\mathbf{e} ; \varepsilon_{0}\right)$ onto its image by $\mathbf{f}$.

As in the case of $K$-fluxes, the vertex $\mathbf{e}$ in (iii) of Definition 1.3 will be called a vertex of coerciveness for the $\Delta^{i_{1}, i_{2}}$-flux $\mathbf{f}$. We denote by $\mathcal{F}^{+}\left(\Delta^{i_{1}, i_{2}}\right)$ the set of $\Delta^{i_{1}, i_{2}}$-fluxes of first kind.

1.4 Definition. We say that $\mathbf{g}: \Delta^{i_{1}, i_{2}} \rightarrow K$ is a $\Delta^{i_{1}, i_{2}}$-flux if there exist $\mathbf{f} \in$ $\mathcal{F}^{+}\left(\Delta^{i_{1}, i_{2}}\right)$ and $S \in \mathcal{S}(K), S=\left[j_{1}, \ldots, j_{p}\right]$, with

$$
\left\{j_{1}, \ldots, j_{p}\right\} \cap\left\{i_{1}, i_{2}\right\}=\emptyset \text { or }\left\{i_{1}, i_{2}\right\},
$$

such that $\mathbf{g}=S \mathbf{f}$.

We denote by $\mathcal{F}\left(\Delta^{i_{1}, i_{2}}\right)$ the set of $\Delta^{i_{1}, i_{2}}$-fluxes.

The first and elementary fact about the above definitions is stated in the following lemma.

1.5 Lemma. Every $\Delta^{i_{1}, i_{2}}$-flux can be extended to a $K$-flux.

Proof. Let $\mathbf{g}$ be a $\Delta^{i_{1}, i_{2}}$-flux, with $\mathbf{g}=S \mathbf{f}, S \in \mathcal{S}(K), \mathbf{f} \in \mathcal{F}^{+}\left(\Delta^{i_{1}, i_{2}}\right)$. For $\mathbf{u} \in K$, $\mathbf{u}=\left(u_{1}, \ldots, u_{n}\right)$, define $\mathbf{u}^{*} \in K, \mathbf{u}^{*}=\left(u_{1}^{*}, \ldots, u_{n}^{*}\right)$ by

$$
u_{i}^{*}= \begin{cases}u_{i}, & \text { if } i \notin\left\{i_{1}, i_{2}\right\}, \\ 1-u_{i_{2}}, & \text { if } i=i_{1}, \\ 1-u_{i_{1}}, & \text { if } i=i_{2} .\end{cases}
$$

The affine map $\mathbf{u} \mapsto \mathbf{u}^{*}$ is a bijection of $K$ onto itself which sends $\Delta^{i_{1}, i_{2}}$ onto $\overline{K-\Delta^{i_{1}, i_{2}}}$ and vice versa. We then extend $\mathbf{g}$ to $K-\Delta^{i_{1}, i_{2}}$ setting

$$
\mathbf{g}(\mathbf{u})=S\left[\mathbf{f}\left(\mathbf{u}^{*}\right)^{*}\right]
$$

for $\mathbf{u} \in K$, with $u_{i_{1}}+u_{i_{2}} \geq 1$. It is easy to see that the new map $\mathbf{g}: K \rightarrow K$, defined out of $\Delta^{i_{1}, i_{2}}$ by (1.4), is a $K$-flux. 
In what follows, frequently, propositions about $K$-fluxes will have corresponding ones about $\Delta^{i_{1}, i_{2}}$-fluxes. We will denote this fact with an expression between parentheses like '(respectively, $\Delta^{i_{1}, i_{2}}$-flux)' just after the assertion for $K$-fluxes.

We remark that we could as well define the prisms $\Delta^{\left(i_{1}, i_{2}\right) \ldots\left(i_{2 k-1}, i_{2 k}\right)}$, with $\bigcap_{j=1}^{k}\left\{i_{2 j-1}, i_{2 j}\right\}=\emptyset, i_{2 j-1}<i_{2 j}$, and $\left\{i_{1}, \ldots, i_{2 k}\right\} \subset\{1, \ldots, n\}$, by

$$
\Delta^{\left(i_{1}, i_{2}\right) \ldots\left(i_{2 k-1}, i_{2 k}\right)}=\left\{\mathbf{u} \in K \mid \quad u_{i_{2 j-1}}+u_{i_{2 j}} \leq 1, \quad j=1, \ldots, k\right\}
$$

and then define the $\Delta^{\left(i_{1}, i_{2}\right) \ldots\left(i_{2 k-1}, i_{2 k}\right)}$-fluxes in analogy to the $\Delta^{i_{1}, i_{2}}$-fluxes. Every result that we obtain in this work for $\Delta^{i_{1}, i_{2}}$-fluxes could be immediately extended to $\Delta^{\left(i_{1}, i_{2}\right) \ldots\left(i_{2 k-1}, i_{2 k}\right)}$-fluxes. For instance, it is immediate to verify that Lemma 1.5 extends to the statement that every $\Delta^{\left(i_{1}, i_{2}\right) \ldots\left(i_{2 k-1}, i_{2 k}\right)}$-flux can be extended to a $K$-flux. Nevertheless, we will content ourselves in referring explicitly only to $\Delta^{i_{1}, i_{2}}$-fluxes, in order to avoid cumbersome notations.

Simple examples of $K$-fluxes are provided by maps $\mathbf{f}=\left(f_{1}, \ldots, f_{n}\right)$ of the form

$$
f_{i}(\mathbf{u})=\frac{a_{i} u_{i}^{p_{i}}}{a_{i} u_{i}^{p_{i}}+\left(1-u_{i}\right) h_{i}(\mathbf{u})}
$$

for $a_{i}, p_{i}$, positive constants and $h_{i}$ nonnegative continuous functions satisfying $h_{i}(\mathbf{u})>0$, if $u_{i}=0, i=1, \ldots, n$. As examples of $\Delta^{1,2}$-fluxes for $n=2$ (we say simply $\Delta$-fluxes in this case), we have the maps $\mathbf{f}=\left(f_{1}, f_{2}\right)$ of the form

$$
\begin{aligned}
& f_{1}(u, v)=\frac{a u^{p}}{a u^{p}+b v^{q}+(1-u-v) h_{1}(u, v)}, \\
& f_{2}(u, v)=\frac{b v^{q}}{a u^{p}+b v^{q}+(1-u-v) h_{2}(u, v)},
\end{aligned}
$$

for $a, b, p, q$ positive constants, and $h_{1}, h_{2}$ nonnegative continuous functions satisfying $h_{1}(0,0)>0, h_{2}(0,0)>0$. The flux functions of the $2 \times 2$ systems arising as Buckley-Leverett models for three-phase flow in porous media can have this form and are always $\Delta$-fluxes (see $[8,3]$ ).

We can combine the above maps to get examples of $\Delta^{i_{1}, i_{2}}$-fluxes for $n>2$. For instance, for $n=3$, the maps $\mathbf{f}=\left(f_{1}, f_{2}, f_{3}\right)$ given by

$$
\begin{aligned}
& f_{1}(u, v, w)=\frac{a u^{p}}{a u^{p}+b v^{q}+(1-u-v) h_{1}(u, v, w)} \\
& f_{2}(u, v, w)=\frac{b v^{q}}{a u^{p}+b v^{q}+(1-u-v) h_{2}(u, v, w)} \\
& f_{3}(u, v, w)=\frac{c w^{r}}{c w^{r}+(1-w) h_{3}(u, v, w)},
\end{aligned}
$$

for $a, b, c, p, q, r$ positive constants and $h_{i}, i=1,2,3$, nonnegative continuous functions satisfying $h_{1}(0,0, w)>0, h_{2}(0,0, w)>0$, and $h_{3}(u, v, 0)>0$, constitute such examples.

Given a $K$-flux (respectively, $\Delta^{i_{1}, i_{2}}$-flux) $\mathbf{g}$, we have $\mathbf{g}=S \mathbf{f}$, with $\mathbf{f} \in \mathcal{F}^{+}(K)$ (respectively, $\mathcal{F}^{+}\left(\Delta^{i_{1}, i_{2}}\right)$ ) and $S \in \mathcal{S}(K)$, which we can denote as in (1.1). So, we will associate to $g$ two projections of $\mathbf{R}^{n}, \pi^{b}: \mathbf{u} \mapsto \mathbf{u}^{b}, \pi^{\sharp}: \mathbf{u} \mapsto \mathbf{u}^{\sharp}$, where $\pi^{b}$ is 
the orthogonal projection of $\mathbf{R}^{n}$ onto the space spanned by $e_{j_{k}}, k=1, \ldots, p$, and $\pi^{\sharp}=I-\pi^{b}$, where $I$ is the identity map.

In this work we will study the initial-boundary value problem given by a system of conservation laws of the form

$$
\frac{\partial}{\partial t} \mathbf{u}+\frac{\partial}{\partial x} \mathbf{g}(\mathbf{u})=0
$$

with $\mathbf{u} \in K$ (respectively, $\left.\Delta^{i_{1}, i_{2}}\right), \mathbf{g} \in \mathcal{F}(K)$ (respectively, $\left.\mathcal{F}\left(\Delta^{i_{1}, i_{2}}\right)\right),(x, t) \in$ $(0, L) \times(0, \infty)$, subjected to the initial and boundary conditions

$$
\begin{aligned}
\mathbf{u}(x, 0) & =\mathbf{u}_{0}(x), \quad x \in(0, L), \\
\mathbf{u}^{b}(x, 0) & =\mathbf{e}^{b}, \\
\mathbf{u}^{\sharp}(L, t) & =\mathbf{e}^{\sharp},
\end{aligned}
$$

with $\mathbf{u}_{0}(x) \in K$ (respectively, $\left.\Delta^{i_{1}, i_{2}}\right)$ and $\mathbf{e}^{b}$, $\mathbf{e}^{\sharp}$ vectors in $\pi^{b}(K), \pi^{\sharp}(K)$, respectively, whose coordinates are equal to zero or one, and such that the vector $\mathbf{e}=\mathbf{e}^{b}+\mathbf{e}^{\sharp}$ is a vertex of coerciveness of the $K$-flux (respectively, $\Delta^{i_{1}, i_{2}}$-flux) $\mathbf{g}$.

Since we do not make any restriction about the eigenvalues of $\nabla \mathbf{g}$ in (1.5), except those which eventually necessarily follow from $\mathrm{g}$ being a $K$-flux (respectively, $\Delta^{i_{1}, i_{2}}$-flux), in general there may exist in $K$ (respectively, $\Delta^{i_{1}, i_{2}}$ ) several umbilic points, that is, points at which some eigenvalues of $\nabla \mathbf{g}$ coincide, submanifolds at which genuine nonlinearity fails, that is, submanifolds where $\nabla \lambda_{i} \cdot \mathbf{r}_{i}=0$, for some eigenvalue $\lambda_{i}$ with associated right eigenvector $\mathbf{r}_{i}$, and also regions where hyperbolicity fails, that is, where some of the eigenvalues of $\nabla \mathbf{g}$ are complex. Because of this, the task of obtaining a weak solution of (1.5)-(1.8), seems to be a very difficult one. Next, we will define what we mean by a measure-valued solution to (1.5)-(1.8), a concept which was introduced (in the context of Cauchy problems) by DiPerna (see [2]).

1.6 Definition. Let $\mathbf{P}(K)$ (respectively, $\mathbf{P}\left(\Delta^{i_{1}, i_{2}}\right)$ ) be the set of probability measures defined on $K$ (respectively, $\Delta^{i_{1}, i_{2}}$ ), and assume $\mathbf{g}$ in (1.5) is a $K$-flux (respectively, $\Delta^{i_{1}, i_{2}}$-flux) with $\mathbf{e}=\mathbf{e}^{\mathrm{b}}+\mathbf{e}^{\sharp}$ a vertex of coerciveness. A measure-valued solution to (1.5)-(1.8) is a measurable map $\nu:(0, L) \times(0,+\infty) \rightarrow \mathbf{P}(K)$ (respectively, $\left.\mathbf{P}\left(\Delta^{i_{1}, i_{2}}\right)\right)$ satisfying:

(i) for each $\phi \in C_{0}^{\infty}([0, L) \times[0, \infty))$ (that is, $\phi \in C_{0}^{\infty}((0, L) \times(0, \infty))$ and $\operatorname{Supp} \phi$ is a compact subset of $[0, L) \times[0, \infty))$ we have

$$
\begin{aligned}
\iint_{[0, L] \times[0, \infty)}\left\{\left\langle\nu_{x, t}, \mathbf{u}^{\mathrm{b}}\right\rangle \phi_{t}+\left\langle\nu_{x, t}, \mathbf{g}^{\mathrm{b}}(\mathbf{u})\right\rangle \phi_{x}\right\} d x d t \\
\quad+\int_{0}^{\infty} \mathbf{e}^{\mathrm{b}} \phi(0, t) d t+\int_{0}^{L} \mathbf{u}_{0}^{\mathrm{b}}(x) \phi(x, 0) d x=0 ;
\end{aligned}
$$

(ii) for each $\psi \in C_{0}^{\infty}((0, L] \times[0, \infty))$ we have

$$
\begin{aligned}
\iint_{[0, L] \times[0, \infty)}\left\{\left\langle\nu_{x, t}, \mathbf{u}^{\sharp}\right\rangle\right. & \psi_{t} \\
& \left.+\left\langle\nu_{x, t}, \mathbf{g}^{\sharp}(\mathbf{u})\right\rangle \psi_{x}\right\} d x d t \\
& -\int_{0}^{\infty}\left(\mathbf{l}^{\sharp}-\mathbf{e}^{\sharp}\right) \psi(0, t) d t+\int_{0}^{L} \mathbf{u}_{0}^{\sharp}(x) \psi(x, 0) d x d t=0,
\end{aligned}
$$


where $\mathbf{l}^{\sharp}=\pi^{\sharp}(\mathbf{l})$ and $\mathbf{l}$ is the vector in $K$ whose coordinates all are equal to 1 (observe that $\mathbf{g}^{b}(\mathbf{u})=\mathbf{e}^{b}$, if $\mathbf{u}^{b}=\mathbf{e}^{b}$, and $\mathbf{g}^{\sharp}(\mathbf{u})=\mathbf{l}^{\sharp}-\mathbf{e}^{\sharp}$, if $\mathbf{u}^{\sharp}=\mathbf{e}^{\sharp}$ );

(iii) for a.e. $(x, t) \in(0, L) \times(0, \infty)$ we have

$$
\operatorname{Supp} \nu_{x, t} \subset K \quad\left(\text { respectively, } \Delta^{i_{1}, i_{2}}\right) .
$$

An important consequence of the above definition is given by the following proposition, which is a key result for the study of the dynamics of the $\mathrm{m}-\mathrm{v}$ solutions of (1.5)-(1.8), as it will be seen further on.

1.7 Proposition. Let $\nu_{x, t}$ be a measure-valued solution to (1.5)-(1.8). Given any $T>0$, for each $\zeta \in C_{0}^{1}([0, T])$ and a.e. $x \in(0, L)$ we have

$$
\begin{aligned}
& \left|\int_{0}^{T}\left(\left\langle\nu_{x, t}, \mathbf{f}^{b}(\mathbf{u})\right\rangle-\mathbf{e}^{b}\right) \zeta(t) d t\right| \leq C x \operatorname{Var}(\zeta), \\
& \left|\int_{0}^{T}\left(\left\langle\nu_{x, t}, \mathbf{f}^{\sharp}(\mathbf{u})\right\rangle-\mathbf{e}^{\sharp}\right) \zeta(t) d t\right| \leq C(L-x) \operatorname{Var}(\zeta),
\end{aligned}
$$

where $C>0$ is a constant independent of $T$ and $x$, and $\mathbf{f} \in \mathcal{F}^{+}(K)$ (respectively, $\left.\mathcal{F}^{+}\left(\Delta^{i_{1}, i_{2}}\right)\right)$ satisfies $\mathbf{g}=S \mathbf{f}, S \in \mathcal{S}(K)$. In particular, we have

$$
\lim _{T \rightarrow \infty} \frac{1}{T} \int_{0}^{T}\left\langle\nu_{x, t}, \mathbf{f}\right\rangle d t=\mathbf{e},
$$

for a.e. $x \in(0, L)$, with a rate of convergence $O\left(T^{-1}\right)$.

Proof. Fix $x_{0} \in(0, L)$ and let $\delta \in C_{0}^{1}((-1,1)), \delta \geq 0$, satisfying $\int_{-1}^{1} \delta(s) d s=1$. Set $\delta_{h}(s)=h^{-1} \delta\left(h^{-1} s\right)$. In (1.9), Definition 1.6, choose $\phi(x, t)=\zeta(t) \chi_{h}^{0}(x)$, where

$$
\chi_{h}^{0}(x)=1-\int_{0}^{x} \delta_{h}\left(s-x_{0}\right) d s,
$$

with $h<\min \left\{x_{0}, L-x_{0}\right\}$, and in (1.10) choose $\psi(x, t)=\zeta(t) \chi_{h}^{L}(x)$, where

$$
\chi_{h}^{L}(x)=\int_{0}^{x} \delta_{h}\left(s-x_{0}\right) d s,
$$

with $h<\min \left\{x_{0}, L-x_{0}\right\}$. Then, if $x_{0}$ is a Lebesgue point of the measurable function (see [9])

$$
\int_{0}^{T}\left\langle\nu_{x, t}, \mathbf{f}(\mathbf{u})\right\rangle \zeta(t) d t
$$

we get that (cf. [6])

$$
\int_{0}^{L} \delta_{h}(x)\left\{\int_{0}^{T}\left\langle\nu_{x, t}, \mathbf{f}(\mathbf{u})\right\rangle \zeta(t) d t\right\} d x \stackrel{h \rightarrow 0}{\longrightarrow} \int_{0}^{T}\left\langle\nu_{x_{0}, t}, \mathbf{f}(\mathbf{u})\right\rangle \zeta(t) d t .
$$

So, letting $h \rightarrow 0$ in (1.9) and (1.10) for $\phi(x, t)=\chi_{h}^{0}(x) \zeta(t)$ and $\psi(x, t)=\chi_{h}^{L}(x) \zeta(t)$, respectively, noting that $\mathbf{f}^{\sharp}(\mathbf{u})=\mathbf{l}^{\sharp}-\mathbf{g}^{\sharp}(\mathbf{u})$, we obtain

$$
\int_{0}^{T}\left\{\left\langle\nu_{x_{0}, t}, \mathbf{f}^{b}(\mathbf{u})\right\rangle-\mathbf{e}^{\mathrm{b}}\right\} \zeta(t) d t=\int_{0}^{x_{0}} \int_{0}^{T}\left\langle\nu_{x, t}, \mathbf{u}^{\mathrm{b}}\right\rangle \zeta^{\prime}(t) d t d x,
$$




$$
\int_{0}^{T}\left\{\left\langle\nu_{x_{0}, t}, \mathbf{f}^{\sharp}(\mathbf{u})\right\rangle-\mathbf{e}^{\sharp}\right\} \zeta(t) d t=\int_{x_{0}}^{L} \int_{0}^{T}\left\langle\nu_{x, t}, \mathbf{u}^{\sharp}\right\rangle \zeta^{\prime}(t) d t d x .
$$

Now, taking modulus on both sides of (1.15), (1.16), using (iii) of Definition 1.6 and making obvious estimates, we get (1.12), (1.13). For (1.14), we observe that (1.12), (1.13) hold, by passing to the limit, for $\zeta(t)=\chi(0, T)(t)$. With this choice for $\zeta$, where we have $\operatorname{Var}(\zeta)=2$, we divide (1.12) and (1.13) by $T$ and let $T \rightarrow \infty$ to get (1.14) with the asserted rate of convergence.

\section{EXISTENCE: VANISHING VISCOSITY METHOD}

We consider the approximation of system (1.5) given by the vanishing viscosity method, that is, we introduce an artificial viscosity term and consider the resulting parabolic system

$$
\frac{\partial}{\partial t} \mathbf{u}+\frac{\partial}{\partial x} \mathbf{g}(\mathbf{u})=\varepsilon \frac{\partial^{2}}{\partial x^{2}} \mathbf{u} .
$$

We assume $\mathbf{g}$ to be a $C^{3} K$-flux (respectively, $\Delta^{i_{1}, i_{2}}$-flux).

The purpose of this section is the proof of the following theorem.

2.1 Theorem. There exists a subsequence of solutions of $(2.1)$ in $(0, L) \times(0, \infty)$, satisfying suitable initial-boundary conditions, which generates, as $\varepsilon \rightarrow 0$, a measure-valued solution to (1.5)-(1.8).

Before proving Theorem 2.1 we want to state a preliminary result about the solutions of initial-boundary value problems for parabolic systems like (2.1). So, let us set the following initial and boundary conditions for system (2.1)

$$
\begin{aligned}
\mathbf{u}(x, 0) & =\mathbf{u}_{0}(x), \quad x \in(0, L), \\
\mathbf{u}^{\mathrm{b}}(0, t) & =\mathbf{u}^{\mathrm{b}}(L, t)=\mathbf{e}^{\mathrm{b}}, \\
\mathbf{u}^{\sharp}(0, t) & =\mathbf{u}^{\sharp}(L, t)=\mathbf{e}^{\sharp},
\end{aligned}
$$

where $\mathbf{u}_{0}(x), \mathbf{e}^{b}, \mathbf{e}^{\sharp}$ are the same as in (1.6)-(1.8).

We will now state a simple but useful lemma on invariant regions for nonlinear parabolic systems, which is in fact an easy corollary of the general result in [1] (see also [10]).

We consider, for the moment, a general initial (or initial-boundary) value problem for system (2.1) with $\mathbf{g}$ a map of class $C^{3},(x, t) \in(a, b) \times(0, \infty)$, where we can eventually have $a=-\infty, b=\infty$, and we assume that there are given appropriate boundary conditions.

Now, let $B \subset \mathbf{R}^{n}$ be given by

$$
B=\bigcap_{i=1}^{N} B_{i},
$$

with

$$
B_{i}=\left\{\mathbf{u} \in \mathbf{R}^{n}: \quad G_{i}(\mathbf{u}) \leq 0\right\},
$$


for certain smooth functions $G_{i}: \mathbf{R}^{n} \rightarrow \mathbf{R}, i=1, \ldots, N$.

We assume that $\mathbf{u}(x, t)$ is a smooth solution of $(2.1)$ in $(a, b) \times(0, T)$, satisfying certain initial (or initial-boundary) conditions, and we also suppose as in [1] that $\mathbf{u}(x, t)$ satisfies:

for each fixed $t$, there exists a compact interval

$I \subset(a, b)$ such that if $x \notin I$, then $\mathbf{u}(x, t) \in \operatorname{int}(B)$.

Condition (2.6) apparently does not allow boundary data taking values on the boundary of $B$. In this case, the usual procedure is to perturb slightly the boundary conditions in order to satisfy condition (2.6), get the invariancy for the new problem and then obtain the invariancy for the original problem by a limit process.

2.2 Lemma. Assume that the functions $G_{i}$ satisfy the quasiconvexity condition

$$
\begin{aligned}
& \xi^{\top} \nabla^{2} G_{i}(\xi) \geq 0, \quad \text { for every } \xi \text { such that }\left\langle\nabla G_{i}, \xi\right\rangle=0, \\
& \text { in } \partial B_{i} \cap \partial B, i=1, \ldots, N,
\end{aligned}
$$

and let the map $\mathbf{g}$ in (2.1) satisfy

$$
T_{\mathbf{g}(\mathbf{u})}\left(\mathbf{g}\left(\partial B_{i}\right)\right) \subset T_{\mathbf{u}}\left(\partial B_{i}\right),
$$

for each $\mathbf{u} \in \partial B_{i} \cap \partial B, i=1, \ldots, N$ (where we consider both affine spaces as translated to the origin). Then $B$ is invariant for the solution of the nonlinear parabolic system (2.1); that is, if the initial and boundary conditions are in $B$, then $\mathbf{u}(x, t) \in B$ for all $(x, t) \in(a, b) \times(0, T)$.

Proof. In order to apply the Chueh-Conley-Smoller theorem on invariant regions [1] we need only to prove the following:

$$
\nabla G_{i} \text { is a left-eigenvector of } \nabla \mathbf{g} \text { on } \partial B_{i} \cap \partial B \text {, for each } i=1, \ldots, N \text {. }
$$

Now, if $\xi \in T_{\mathbf{u}}\left(\partial B_{i}\right)$, with $\mathbf{u} \in \partial B_{i} \cap \partial B$, then $\nabla \mathbf{g} \cdot \xi \in T_{\mathbf{g}(\mathbf{u})}\left(\mathbf{g}\left(\partial B_{i}\right)\right)$. By hypotheses (2.8) we have that, since $\nabla G_{i}(\mathbf{u})$ is normal to $T_{\mathbf{u}}\left(\partial B_{i}\right)$, then it is also normal to $T_{\mathbf{g}(\mathbf{u})}\left(\mathbf{g}\left(\partial B_{i}\right)\right)$, if $\mathbf{u} \in \partial B_{i} \cap \partial B, i=1, \ldots, N$. So, we have,

$$
\left\langle\nabla \mathbf{g}(\mathbf{u})^{\top} \cdot \nabla G_{i}(\mathbf{u}), \xi\right\rangle=\left\langle\nabla G_{i}(\mathbf{u}), \nabla \mathbf{g}(\mathbf{u}) \xi\right\rangle=0,
$$

for all $\xi \in T_{\mathbf{u}}\left(\partial B_{i}\right), \mathbf{u} \in \partial B_{i} \cap \partial B, i=1, \ldots, N$. Then,

$$
\nabla \mathbf{g}(\mathbf{u})^{\top} \nabla G_{i}(\mathbf{u}) \| \nabla G_{i}(\mathbf{u})
$$

that is, there exists a certain $\mu_{i} \in \mathbf{R}, \mu_{i}=\mu_{i}(\mathbf{u})$, such that

$$
\nabla \mathbf{g}(\mathbf{u})^{\top} \nabla G_{i}(\mathbf{u})=\mu_{i} \nabla G_{i}(\mathbf{u})
$$

and so, we have (2.9) and the lemma is proved. 
Proof of Theorem 2.1. We will prove that, for each $\varepsilon>0$, there exists a solution $\mathbf{u}^{\varepsilon}(x, t)$ of problem (2.1)-(2.4) defined for all $(x, t) \in[0, L] \times[0, \infty)$ and taking values in $K$ (respectively, $\Delta^{i_{1}, i_{2}}$ ). Then, Tartar's theorem on the existence of Young measures (see [11]) will give us the existence of a measurable map $\nu:[0, L] \times[0, \infty) \rightarrow \mathbf{P}(K)$ (respectively, $\mathbf{P}\left(\Delta^{i_{1}, i_{2}}\right)$ ), which we denote by $\nu_{x, t}$, such that $\operatorname{Supp} \nu_{x, t} \subset K$ (respectively, $\left.\Delta^{i_{1}, i_{2}}\right)$ and for all $h \in C(K)$ (respectively, $\left.C\left(\Delta^{i_{1}, i_{2}}\right)\right)$ we have

$$
w^{*}-\lim h\left(\mathbf{u}^{\varepsilon}(x, t)\right)=\left\langle\nu_{x, t}, h(\mathbf{u})\right\rangle,
$$

a.e. $(x, t) \in(0, L) \times(0, \infty)$.

Then, after proving the existence of $\mathbf{u}^{\varepsilon}(x, t)$ as above, we prove that the associated Young measure $\nu_{x, t}$ satisfies (1.9), (1.10) of Definition 1.6, and this will conclude the proof of the Theorem 2.1.

We begin by recalling the solution of the elementary initial-boundary value problem for the heat equation:

$$
\begin{aligned}
\frac{\partial}{\partial t} v & =\varepsilon \frac{\partial^{2}}{\partial x^{2}} v, \\
v(x, 0) & =v_{0}(x), \\
v(0, t) & =v(L, t)=0 .
\end{aligned}
$$

Its solution can be represented in the form

$$
v(x, t)=\int_{0}^{L} G(x, y, t) v_{0}(y) d y,
$$

with

$$
G(x, y, t)=\frac{1}{\sqrt{4 \pi \varepsilon t}} \sum_{s \in \mathbf{Z}}\left(e^{-(x-y-2 s L)^{2} / 4 \varepsilon t}-e^{-(x+y-2 s L)^{2} / 4 \varepsilon t}\right) .
$$

Similarly, the solution of the problem given by (2.11), (2.12) and

$$
v(0, t)=v(L, t)=1,
$$

can be expressed by

$$
\tilde{v}(x, t)=1+\int_{0}^{L} G(x, y, t)\left(v_{0}(y)-1\right) d y .
$$

Let us identify any function $h(x, t)$ defined in $[0, L] \times[0, \infty)$ with a map $t \mapsto h(t)$ from $[0, \infty)$ to the space of functions defined in $[0, L]$. Also, let us denote by $T_{G(\tau)} h(t)$ the function defined in $[0, L]$ by

$$
\left[T_{G(\tau)} h(t)\right](x)=\int_{0}^{L} G(x, y, \tau) h(y, t) d y,
$$

and similarly for $T_{G_{y}(\tau)} h(t)$, where $G_{y}=\frac{\partial}{\partial y} G$. So, a smooth solution $\mathbf{u}^{\varepsilon}(x, t)$ to (2.1)-(2.4), defined for $(x, t) \in[0, L] \times[0, T]$, satisfies the integral equation

$$
\mathbf{u}^{\varepsilon}(t)=\mathbf{e}+T_{G(t)}\left(\mathbf{u}_{0}-\mathbf{e}\right)-\int_{0}^{t} T_{G_{y}(t-s)} \mathbf{g}\left(\mathbf{u}^{\varepsilon}(s)\right) d s .
$$


Now, we define the operator $\mathbf{v} \mapsto \mathcal{L}(\mathbf{v})$, for $\mathbf{v} \in L^{\infty}\left([0, L] \times[0, \infty) ; \mathbf{R}^{n}\right)$, by

$$
\mathcal{L}(\mathbf{v})(t)=\mathbf{e}+T_{G(t)}\left(\mathbf{u}_{0}-\mathbf{e}\right)-\int_{0}^{t} T_{G_{y}(t-s)} \mathbf{g}(\mathbf{v}(s)) d s .
$$

By standard arguments (see [4]) we prove that this operator has a fixed point in $L^{\infty}\left([0, L] \times[0, T] ; \mathbf{R}^{n}\right)$ for $T$ sufficiently small, and that this fixed point has suitable regularity properties. We then prove, again by standard arguments (see [4]), that this fixed point $\mathbf{u}^{\varepsilon}(x, t)$ is a local solution to (2.1)-(2.4). Now, we apply Lemma 2.2. It is easy to verify that $K$ (respectively, $\Delta^{i_{1}, i_{2}}$ ) and $\mathbf{g}$, being a $C^{3}$ $K$-flux (respectively, $\Delta^{i_{1}, i_{2}}$-flux), satisfy the hypotheses (2.7), (2.8) of Lemma 2.2, where $G_{i}$ are the obvious affine functions defining $K$ (respectively, $\Delta^{i_{1}, i_{2}}$ ). So, $K$ (respectively, $\Delta^{i_{1}, i_{2}}$ ) is an invariant region for the solution of the problem (2.1)(2.4). Hence, we can easily extend the local solution $\mathbf{u}^{\varepsilon}(x, t)$ to a global solution, that is, a smooth solution of $(2.1)-(2.4)$ defined in $[0, L] \times[0, \infty)$. This global solution $\mathbf{u}^{\varepsilon}(x, t)$ takes its values in $K$ (respectively, $\left.\Delta^{i_{1}, i_{2}}\right)$.

Let $\nu_{x, t}$ be a Young measure associated to a subsequence of $\mathbf{u}^{\varepsilon}(x, t)$, when $\varepsilon \rightarrow 0$. We will now prove that $\nu_{x, t}$ satisfies (1.9), (1.10) in Definition 1.6.

By $(2.10)$, we have that, for every $\phi, \psi \in C_{0}^{\infty}((0, L) \times[0, \infty))$

$$
\begin{gathered}
\iint_{(0, L) \times(0, \infty)}\left\{\left\langle\nu_{x, t}, \mathbf{u}^{b}\right\rangle \phi_{t}+\left\langle\nu_{x, t}, \mathbf{g}^{\mathrm{b}}(\mathbf{u})\right\rangle \phi_{x}\right\} d x d t \\
+\int_{0}^{L} \mathbf{u}_{0}^{\mathrm{b}}(x) \phi(x, 0) d x=0, \\
\iint_{(0, L) \times(0, \infty)}\left\{\left\langle\nu_{x, t}, \mathbf{u}^{\sharp}\right\rangle \psi_{t}+\left\langle\nu_{x, t}, \mathbf{g}^{\sharp}(\mathbf{u})\right\rangle \psi_{x}\right\} d x d t \\
+\int_{0}^{L} \mathbf{u}_{0}^{\sharp}(x) \psi(x, 0) d x=0 .
\end{gathered}
$$

Let $\left\{j_{1}, \ldots, j_{p}\right\}$ be the set related with $\mathbf{g}$ by $\mathbf{g}=S \mathbf{f}, \mathbf{f} \in \mathcal{F}^{+}(K)$ (respectively, $\left.\mathcal{F}^{+}\left(\Delta^{i_{1}, i_{2}}\right)\right)$ and $S \in \mathcal{S}(K), S=\left[j_{1}, \ldots, j_{p}\right]$. First, we consider $i \in\left\{j_{1}, \ldots, j_{p}\right\}$. We have $\left\langle\mathbf{e}^{b}, \mathbf{e}_{i}\right\rangle=0$ or 1 , where $\mathbf{e}_{i}$ is the $i$-th element of the canonical basis of $\mathbf{R}^{n}$. Initially, we assume that $\left\langle\mathbf{e}^{b}, \mathbf{e}_{i}\right\rangle=0$. We will prove, in this case, that, for every $\phi \in C_{0}^{\infty}([0, L) \times[0, \infty))$, we have

$$
\iint_{(0, L) \times[0, \infty)}\left\{\left\langle\nu_{x, t}, u_{i}\right\rangle \phi_{t}+\left\langle\nu_{x, t}, g_{i}(\mathbf{u})\right\rangle \phi_{x}\right\} d x d t+\int_{0}^{L} u_{0 i}(x) \phi(x, 0) d x=0 .
$$

Observe that for $\phi$ in (2.19) we can have Supp $\phi \cap\{x=0\} \neq \emptyset$. Now, for $\phi \in$ $C_{0}^{\infty}([0, L) \times[0, \infty))$, with $\phi \geq 0$ and $\phi_{x}(0, t) \equiv 0$, we have

$$
\begin{aligned}
\varepsilon \iint\left(u_{i}^{\varepsilon}\right)_{x x} \phi d x d t=-\varepsilon \int_{0}^{\infty}\left(u_{i}^{\varepsilon}\right)_{x}(0, t) \phi & (0, t) d t \\
& +\varepsilon \iint u_{i}^{\varepsilon} \phi_{x x} d x d t \leq \varepsilon \iint u_{i}^{\varepsilon} \phi_{x x} d x d t
\end{aligned}
$$

since $\left(u_{i}^{\varepsilon}\right)_{x}(0, t) \geq 0$, for all $t>0$. So, for every $\phi \in C_{0}^{\infty}([0, L) \times[0, \infty))$, with $\phi \geq 0, \phi_{x}(0, t) \equiv 0$, we have

$$
\iint\left\{\left\langle\nu_{x, t}, u_{i}\right\rangle \phi_{t}+\left\langle\nu_{x, t}, f_{i}(\mathbf{u})\right\rangle \phi_{x}\right\} d x d t+\int_{0}^{L} u_{0 i}(x) \phi(x, 0) d x \geq 0 .
$$


Now, taking $\phi(x, t)=\zeta(t) \chi^{n}(x)$, with $\zeta \in C_{0}^{\infty}((0, \infty)), \zeta \geq 0$, and $\chi^{n} \in C_{0}^{\infty}([0, L))$ satisfying $\chi^{n}(x)=1$, for $0 \leq x \leq \frac{x_{0}}{2}, 0 \leq \chi^{n} \leq 1, \chi^{n} \rightarrow \chi_{\left[0, x_{0}\right]}$ (the characteristic function of $\left[0, x_{0}\right]$ ), as $n \rightarrow \infty$, with $x_{0} \in[0, L$ ) (a sequence of this type was constructed in Proposition 1.7), we get

$$
0 \leq \int_{0}^{\infty}\left\langle\nu_{x_{0}, t}, f_{i}(\mathbf{u})\right\rangle \zeta(t) d t \leq C \operatorname{Var}(\zeta) x_{0},
$$

for some fixed constant $C>0$, provided $x_{0}$ is a Lebesgue point of the function (cf. [6])

$$
\int_{0}^{\infty}\left\langle\nu_{x, t}, f_{i}(\mathbf{u})\right\rangle \zeta(t) d t
$$

So, we have

$$
\lim _{x_{\circ} \rightarrow 0} \int_{0}^{\infty}\left\langle\nu_{x_{0}, t}, f_{i}(\mathbf{u})\right\rangle \zeta(t) d t=0,
$$

for all $\zeta \in C_{0}^{\infty}((0, \infty))$, with $\zeta \geq 0$, which gives, by linearity and density, (2.22) for all $\zeta \in C_{0}^{1}([0, \infty))$.

An analogous procedure would give us

$$
\lim _{x_{0} \rightarrow 0} \int_{0}^{\infty}\left\langle\nu_{x_{\circ}, t}, f_{i}(\mathbf{u})\right\rangle \zeta(t) d t=\int_{0}^{\infty} \zeta(t) d t,
$$

for all $\zeta \in C_{0}^{1}\left([0, \infty)\right.$ ), if we had $\left\langle\mathbf{e}^{b}, \mathbf{e}_{i}\right\rangle=1$ (in this case we use the fact that $\left(u_{i}^{\varepsilon}\right)_{x}(0, t) \leq 0$, for all $\left.t>0\right)$.

Now, let $\phi \in C_{0}^{1}([0, L) \times[0, \infty))$ and set

$$
\begin{array}{ll}
\phi_{\delta}(x, t)=\phi(x-\delta, t), & \text { for } x \geq \delta, \\
\phi_{\delta}(x, t)=0, & \text { for } x<\delta .
\end{array}
$$

We approximate $\phi_{\delta}$ by $\phi_{\delta}^{n} \in C_{0}^{1}((0, L) \times[0, \infty))$, setting $\phi_{\delta}^{n}=\phi_{\delta} \chi^{n}$, with $\chi^{n} \in$ $C_{0}^{\infty}((0, L)), 0 \leq \chi^{n} \leq 1$, Supp $\chi^{n} \subset[\delta, L]$ and $\chi^{n} \rightarrow \chi_{[\delta, L]}$, as $n \rightarrow \infty$. Substituting $\phi$ by $\phi_{\delta}^{n}$ in $\left(2.18^{b}\right)$, assuming that $\delta$ is a Lebesgue point of the function

$$
\int_{0}^{\infty}\left\langle\nu_{x, t}, \mathbf{g}^{b}(\mathbf{u})\right\rangle d t
$$

we get

$$
\begin{aligned}
\iint_{(\delta, L) \times(0, \infty)}\left\{\left\langle\nu_{x, t},\right.\right. & \left.\left.\mathbf{u}^{\mathrm{b}}\right\rangle\left(\phi_{\delta}\right)_{t}+\left\langle\nu_{x, t}, \mathbf{g}^{\mathrm{b}}(\mathbf{u})\right\rangle\left(\phi_{\delta}\right)_{x}\right\} d x d t \\
& +\int_{0}^{L} \mathbf{u}_{0}^{\mathrm{b}}(x) \phi(x, 0) d x+\int_{0}^{\infty}\left\langle\nu_{\delta, t}, \mathbf{g}^{\mathrm{b}}(\mathbf{u})\right\rangle \phi(0, t) d t=0 .
\end{aligned}
$$

So, making $\delta \rightarrow 0$, and using (2.22), (2.23), we get (1.9) of Definition 1.6.

An entirely analogous procedure led us to (1.10) of Definition 1.6. The theorem is then proved. 


\section{Existence: Finite DifferenCe SCHEMES}

In this section we prove the existence of measure-valued solution to (1.5)-(1.8) using finite difference schemes. We assume that $\mathbf{g}$ in (1.5) is a $C^{2} K$-flux (respectively, $\Delta^{i_{1}, i_{2}}$-flux).

Let $\triangle x, \triangle t$ be the mesh lenghts and $M \in \mathbf{N}$ such that $L=M \triangle x$. Assume to be satisfied the following condition

$$
\frac{\triangle x}{\triangle t} \geq \Lambda \stackrel{\text { def }}{=} \sup _{\substack{i=1, \ldots, n \\ \mathbf{u} \in K\left(\text { respectively, }, \Delta^{i_{1}, i_{2}}\right)}}\left|\nabla g_{i}(\mathbf{u})\right| .
$$

We propose the following scheme in order to generate a m-v solution to (1.5)-(1.8):

$$
\begin{aligned}
& \left(\mathbf{u}^{b}\right)^{0, \tau}=\mathbf{e}^{b}, \quad \tau \in \mathbf{N}, \\
& \left(\mathbf{u}^{\sharp}\right)^{M, \tau}=\mathbf{e}^{\sharp}, \quad \tau \in \mathbf{N}, \\
& \mathbf{u}^{\rho, 0}=\mathbf{u}_{0}(\rho \triangle x), \quad \rho \in \mathbf{N}, 1 \leq \rho \leq M-1,
\end{aligned}
$$

and

$$
\left(\mathbf{u}^{b}\right)^{\rho, \tau+1}=\left(\mathbf{u}^{b}\right)^{\rho, \tau}-\frac{\triangle t}{\triangle x}\left(\mathbf{g}^{\mathrm{b}}\left(\mathbf{u}^{\rho, \tau}\right)-\mathbf{g}^{\mathrm{b}}\left(\mathbf{u}^{\rho-1, \tau}\right)\right),
$$

$\tau, \rho \in \mathbf{N}, 1 \leq \rho \leq M$,

$$
\left(\mathbf{u}^{\sharp}\right)^{\rho, \tau+1}=\left(\mathbf{u}^{\sharp}\right)^{\rho, \tau}-\frac{\triangle t}{\triangle x}\left(\mathbf{g}^{\sharp}\left(\mathbf{u}^{\rho+1, \tau}\right)-\mathbf{g}^{\sharp}\left(\mathbf{u}^{\rho, \tau}\right)\right),
$$

$\tau, \rho \in \mathbf{N}, 0 \leq \rho \leq M-1$.

First, we consider $i \in\left\{j_{1}, \ldots, j_{p}\right\}$ and prove that

$$
0 \leq u_{i}^{\rho, \tau} \leq 1, \quad \tau, \rho \in \mathbf{N}, 1 \leq \rho \leq M .
$$

In the case where $\mathbf{g}$ is a $\Delta^{i_{1}, i_{2}}$-flux, we assume first that $\left\{i_{1}, i_{2}\right\} \subset\left\{j_{1}, \ldots, j_{p}\right\}$ and prove, besides (3.7), that

$$
u_{i_{1}}^{\rho, \tau}+u_{i_{2}}^{\rho, \tau} \leq 1, \quad \tau, \rho \in \mathbf{N}, 0 \leq \rho \leq M .
$$

The proof of (3.7), (3.8) is made by induction in $\tau$ and is a consequence of the definition of a $K$-flux (respectively, $\Delta^{i_{1}, i_{2}}$-flux), condition (3.1) and the Mean Value Theorem applied to suitable functions.

Indeed, we have

$$
\begin{aligned}
u_{i}^{\rho, \tau+1}= & u_{i}^{\rho, \tau}-\frac{\triangle t}{\triangle x}\left(g_{i}\left(\mathbf{u}^{\rho, \tau}\right)-g_{i}\left(\mathbf{u}^{\rho-1, \tau}\right)\right) \\
= & u_{i}^{\rho, \tau}-\frac{\triangle t}{\triangle x}\left(g_{i}\left(\mathbf{u}^{\rho, \tau}-u_{i}^{\rho, \tau} \mathbf{e}_{i}\right)-g_{i}\left(\mathbf{u}^{\rho-1, \tau}\right)\right) \\
& -\frac{\triangle t}{\triangle x}\left(g_{i}\left(\mathbf{u}^{\rho, \tau}\right)-g_{i}\left(\mathbf{u}^{\rho, \tau}-u_{i}^{\rho, \tau} \mathbf{e}_{i}\right)\right) \\
= & u_{i}^{\rho, \tau}+\frac{\triangle t}{\triangle x} g_{i}\left(\mathbf{u}^{\rho-1, \tau}\right) \\
& -\frac{\triangle t}{\triangle x}\left(g_{i}\left(\mathbf{u}^{\rho, \tau}\right)-g_{i}\left(\mathbf{u}^{\rho, \tau}-u_{i}^{\rho, \tau} \mathbf{e}_{i}\right)\right) \\
\geq & u_{i}^{\rho, \tau}-\frac{\triangle t}{\triangle x} \Lambda u_{i}^{\rho, \tau} \geq 0
\end{aligned}
$$


if $\mathbf{u}^{\rho, \tau}, \mathbf{u}^{\rho-1, \tau} \in K$, by (3.1), where we have used the fact that $g_{i}\left(\mathbf{u}-u_{i} \mathbf{e}_{i}\right)=0$, $\mathbf{u} \in K, i=1, \ldots, n$. In just the same way, we prove that

$$
1-u_{i}^{\rho, \tau+1} \geq 0, \quad \text { i.e., } \quad u_{i}^{\rho, \tau+1} \leq 1,
$$

if $\mathbf{u}^{\rho, \tau}, \mathbf{u}^{\rho-1, \tau} \in K, i=1, \ldots, n\left(i \neq i_{1}, i_{2}\right.$ in the case of $\Delta^{i_{1}, i_{2}}$-flux $)$.

In the case where $\mathbf{g}$ is a $\Delta^{i_{1}, i_{2}}$-flux and $\left\{i_{1}, i_{2}\right\} \subset\left\{j_{1}, \ldots, j_{p}\right\}$ we begin with the following relation obtained by adding

$$
\begin{array}{r}
u_{i_{1}}^{\rho, \tau+1}+u_{i_{2}}^{\rho, \tau+1}=u_{i_{1}}^{\rho, \tau}+u_{i_{2}}^{\rho, \tau}-\frac{\triangle t}{\triangle x}\left(\left(g_{i_{1}}\left(\mathbf{u}^{\rho, \tau}\right)+g_{i_{2}}\left(\mathbf{u}^{\rho, \tau}\right)\right)\right. \\
\left.-\left(g_{i_{1}}\left(\mathbf{u}^{\rho-1, \tau}\right)+g_{i_{2}}\left(\mathbf{u}^{\rho-1, \tau}\right)\right)\right) .
\end{array}
$$

Then, we consider the line $\mathbf{u}^{\rho, \tau}(t)$ given by

$$
u_{i}^{\rho, \tau}= \begin{cases}u_{i}^{\rho, \tau}, & i \notin\left\{i_{1}, i_{2}\right\}, \\ u_{i_{1}}^{\rho, \tau}+\frac{t}{2}\left(1-u_{i_{1}}^{\rho, \tau}-u_{i_{2}}^{\rho, \tau}\right), & i=i_{1}, \\ u_{i_{2}}^{\rho, \tau}+\frac{t}{2}\left(1-u_{i_{1}}^{\rho, \tau}-u_{i_{2}}^{\rho, \tau}\right), & i=i_{2} .\end{cases}
$$

We set

$$
h(t)=g_{i_{1}}\left(\mathbf{u}^{\rho, \tau}(t)\right)+g_{i_{2}}\left(\mathbf{u}^{\rho, \tau}(t)\right) .
$$

We observe that

$$
h(0)=g_{i_{1}}\left(\mathbf{u}^{\rho, \tau}\right)+g_{i_{2}}\left(\mathbf{u}^{\rho, \tau}\right), \quad h(1)=1,
$$

since $u_{i_{1}}(1)+u_{i_{2}}(1)=1$, and

$$
h^{\prime}(t)=\frac{1}{2}\left(1-u_{i_{1}}-u_{i_{2}}\right) \sum_{j, k=1}^{2} \frac{\partial}{\partial u_{i_{k}}} g_{i_{j}}\left(\mathbf{u}^{\rho, \tau}(t)\right) .
$$

So, using (3.9) and applying the Mean Value Theorem to $h(t)$ we get

$$
\begin{aligned}
u_{i_{1}}^{\rho, \tau+1}+u_{i_{2}}^{\rho, \tau+1}= & u_{i_{1}}^{\rho, \tau}+u_{i_{2}}^{\rho, \tau}-\frac{\triangle t}{\triangle x}\left(h(1)-\left(g_{i_{1}}\left(\mathbf{u}^{\rho-1, \tau}\right)+g_{i_{2}}\left(\mathbf{u}^{\rho-1, \tau}\right)\right)\right) \\
& -\frac{\triangle t}{\triangle x}(h(1)-h(0)) \\
\leq & u_{i_{1}}^{\rho, \tau}+u_{i_{2}}^{\rho, \tau}+\frac{\triangle t}{\triangle x}\left(1-g_{i_{1}}\left(\mathbf{u}^{\rho-1, \tau}\right)-g_{i_{2}}\left(\mathbf{u}^{\rho-1, \tau}\right)\right) \\
& +\Lambda \frac{\triangle t}{\triangle x}\left(1-u_{i_{1}}^{\rho, \tau}-u_{i_{2}}^{\rho, \tau}\right) \\
\leq & 1-\left(1-\Lambda \frac{\triangle t}{\triangle x}\right)\left(1-u_{i_{1}}^{\rho, \tau}-u_{i_{2}}^{\rho, \tau}\right) \leq 1,
\end{aligned}
$$

by (3.1), if $\mathbf{u}^{\rho, \tau}, \mathbf{u}^{\rho-1, \tau} \in \Delta^{i_{1}, i_{2}}$.

The same procedures lead us to analogous results when $i \notin\left\{j_{1}, \ldots, j_{p}\right\}$ and, in the case where $\mathbf{g}$ is a $\Delta^{i_{1}, i_{2}}$-flux, when $\left\{i_{1}, i_{2}\right\} \cap\left\{j_{1}, \ldots, j_{p}\right\}=\emptyset$. So, the 
scheme given by (3.2)-(3.6), with condition (3.1), defines $\mathbf{u}^{\rho, \tau}$ in such a way that $\mathbf{u}^{\rho, \tau} \in K$ (respectively, $\Delta^{i_{1}, i_{2}}$ ), for all $\rho, \tau \in \mathbf{N}, 0 \leq \rho \leq M$. We then define, for $\varepsilon=\triangle x=\Lambda \triangle t$

$$
\begin{aligned}
& \mathbf{u}^{\varepsilon}(x, t)=\mathbf{u}^{\rho, \tau}, \quad \text { if } \quad\left(\rho-\frac{1}{2}\right) \triangle x \leq x<\left(\rho+\frac{1}{2}\right) \triangle x, \\
& \tau \triangle t \leq t<(\tau+1) \triangle t,
\end{aligned}
$$

for all $\rho, \tau \in \mathbf{N}, 0 \leq \rho \leq M$.

So, by Tartar's theorem on the existence of Young measures (see [11]), we can obtain $\nu_{x, t}$, a Young measure associated to a subsequence of $\mathbf{u}^{\varepsilon}(x, t)$, still denoted by $\mathbf{u}^{\varepsilon}(x, t)$, satisfying $\operatorname{Supp} \nu_{x, t} \subset K$ (respectively, $\left.\Delta^{i_{1}, i_{2}}\right)$. Hence, for all $h \in C(K)$ we have (2.10).

Now, by the same proof of the theorem in section 1 of [7], we obtain that for all $\phi, \psi \in C_{0}^{\infty}((0, L) \times[0, \infty))$ we have that equations $(2.18)$ hold for $\nu_{x, t}$. To prove that $\nu_{x, t}$ satisfies (1.9), (1.10) of Definition 1.6 we make use of the following lemma.

3.1 Lemma. Given $\zeta \in C_{0}^{1}((0, T))$ we have

$$
\left|\int_{0}^{T}\left(\mathbf{g}\left(\mathbf{u}^{\varepsilon}(x, t)\right)-\mathbf{g}\left(\mathbf{u}^{\varepsilon}(y, t)\right)\right) \zeta(t) d t\right| \leq C \operatorname{Var}(\zeta)(|x-y|+\varepsilon),
$$

for a.e. $0<x, y<L$, and

$$
\begin{gathered}
\left|\int_{0}^{T}\left(\mathbf{g}^{\mathrm{b}}\left(\mathbf{u}^{\varepsilon}(x, t)\right)-\mathbf{e}^{\mathrm{b}}\right) \zeta(t) d t\right| \leq C \operatorname{Var}(\zeta)(x+\varepsilon), \\
\left|\int_{0}^{T}\left(\mathbf{g}^{\sharp}\left(\mathbf{u}^{\varepsilon}(x, t)\right)-\left(\mathbf{l}^{\sharp}-\mathbf{e}^{\sharp}\right)\right) \zeta(t) d t\right| \leq C \operatorname{Var}(\zeta)(L-x+\varepsilon),
\end{gathered}
$$

for a.e. $0<x<L$ and some constant $C>0$ independent of $x, T$.

Proof. Inequalities (3.11)-(3.13) are consequences of the following two equations.

$$
\begin{aligned}
& \left|\int_{0}^{T}\left(\mathbf{g}^{\mathrm{b}}\left(\mathbf{u}^{\varepsilon}(x, t)\right)-\mathbf{g}^{\mathrm{b}}\left(\mathbf{u}^{\varepsilon}(y, t)\right)\right) \zeta(t) d t\right| \leq C \operatorname{Var}(\zeta)(|x-y|+\varepsilon), \\
& \left|\int_{0}^{T}\left(\mathbf{g}^{\sharp}\left(\mathbf{u}^{\varepsilon}(x, t)\right)-\mathbf{g}^{\sharp}\left(\mathbf{u}^{\varepsilon}(y, t)\right)\right) \zeta(t) d t\right| \leq C \operatorname{Var}(\zeta)(|x-y|+\varepsilon),
\end{aligned}
$$

for a.e. $0<x, y<L$ and some constant $C>0$ independent of $x, y, T$. We will prove (3.14), the proof of (3.15) being entirely analogous. Since, for each $t>0$, $\mathbf{u}^{\varepsilon}(x, t)$ is constant for $\left(\rho-\frac{1}{2}\right) \triangle x \leq x<\left(\rho+\frac{1}{2}\right) \triangle x, \rho \in \mathbf{N}, 0 \leq \rho \leq M$, we can assume that $x=\rho_{1} \triangle x$ and $y=\rho_{2} \triangle x$, with $0 \leq \rho_{1}<\rho_{2} \leq M$. We can also assume 
$T=N \triangle t$, because of the form of the right-hand members of (3.14), (3.15). We then have

$$
\begin{aligned}
\mid \int_{0}^{N \triangle t} & \left(\mathbf{g}^{\mathbf{b}}\left(\mathbf{u}^{\varepsilon}\left(\rho_{1} \triangle x, t\right)\right)-\mathbf{g}^{\mathrm{b}}\left(\mathbf{u}^{\varepsilon}\left(\rho_{2} \triangle x, t\right)\right)\right) \zeta(t) d t \mid \\
\leq & \left|\sum_{\tau=0}^{N-1}\left(\mathbf{g}^{\mathrm{b}}\left(\mathbf{u}^{\rho_{1}, \tau}\right)-\mathbf{g}^{\mathrm{b}}\left(\mathbf{u}^{\rho_{2}, \tau}\right)\right) \zeta(\tau \triangle t) \triangle t\right|+C_{1} \operatorname{Var}(\zeta) \triangle t \\
= & \left|\sum_{\tau=0}^{N-1} \zeta(\tau \triangle t) \sum_{\rho=\rho_{1}+1}^{\rho_{2}}\left(\mathbf{g}^{\mathrm{b}}\left(\mathbf{u}^{\rho, \tau}\right)-\mathbf{g}^{\mathrm{b}}\left(\mathbf{u}^{\rho-1, \tau}\right)\right) \triangle t\right|+C_{1} \operatorname{Var}(\zeta) \triangle t \\
= & \left|\sum_{\tau=0}^{N-1} \zeta(\tau \triangle t) \sum_{\rho=\rho_{1}+1}^{\rho_{2}}\left(\left(\mathbf{u}^{\mathrm{b}}\right)^{\rho, \tau+1}-\left(\mathbf{u}^{\mathrm{b}}\right)^{\rho, \tau}\right) \triangle x\right|+C_{1} \operatorname{Var}(\zeta) \triangle t \\
\leq & \left|\sum_{\rho=\rho_{1}+1}^{\rho_{2}} \sum_{\tau=1}^{N}\left(\mathbf{u}^{\mathrm{b}}\right)^{\rho, \tau}(\zeta((\tau-1) \triangle t)-\zeta(\tau \triangle t)) \triangle x\right|+C_{2} \operatorname{Var}(\zeta) \triangle t \\
\leq & C \operatorname{Var}(\zeta)\left(\left(\rho_{2}-\rho_{1}\right) \triangle x+\varepsilon\right),
\end{aligned}
$$

and this gives (3.14). The lemma is then proved.

Now, (3.11) allows us to obtain a subsequence of $\mathbf{u}^{\varepsilon}(x, t)$, such that, for a.e. $x \in$ $(0, L), \mathbf{g}\left(\mathbf{u}^{\varepsilon}(x, \cdot)\right)$ converges weakly as a function of $t$ (we get first the convergence for a countable dense subset of $(0, L)$ and then use (3.11) to extend the convergence to a.e. $x \in(0, L))$. This weak limit must then coincide a.e. in $(0, L) \times[0, \infty)$ with $\left\langle\nu_{x, t}, \mathbf{g}(\mathbf{u})\right\rangle$. In particular, (3.12), (3.13) gives us

$$
\begin{gathered}
\left|\int_{0}^{T}\left(\left\langle\nu_{x, t}, \mathbf{g}^{\mathbf{b}}(\mathbf{u})\right\rangle-\mathbf{e}^{\mathrm{b}}\right) \zeta(t) d t\right| \leq C \operatorname{Var}(\zeta) x, \\
\left|\int_{0}^{T}\left(\left\langle\nu_{x, t}, \mathbf{g}^{\sharp}(\mathbf{u})\right\rangle-\left(\mathbf{l}^{\sharp}-\mathbf{e}^{\sharp}\right)\right) \zeta(t) d t\right| \leq C \operatorname{Var}(\zeta)(L-x),
\end{gathered}
$$

for a.e. $x \in(0, L)$ and some constant $C>0$ independent of $x, T$. We now use (2.18), (3.16), (3.17) to conclude that $\nu_{x, t}$ satisfies (1.9), (1.10) exactly as we did in the end of the last section. So, $\nu_{x, t}$ constucted by the finite difference scheme given by (3.2)-(3.6), with condition (3.1), is a m-v solution of (1.5)-(1.8).

We conclude this section with the description of another scheme which also gives us a m-v solution to problem (1.5)-(1.8). This time we discretize only the space variable. So, we set

$$
\begin{gathered}
\left(\mathbf{u}^{b}\right)^{0}(t)=\mathbf{e}^{b}, \\
\left(\mathbf{u}^{\sharp}\right)^{M}(t)=\mathbf{e}^{\sharp}, \\
\mathbf{u}^{\rho}(0)=\mathbf{u}_{0}(\rho \triangle x), \quad\left(\mathbf{u}^{\sharp}\right)^{0}(0)=\mathbf{u}_{0}^{\sharp}(0), \quad\left(\mathbf{u}^{b}\right)^{M}(0)=\mathbf{u}_{0}^{b}(L),
\end{gathered}
$$

$\rho \in \mathbf{N}, 1 \leq \rho \leq M-1$, and

$$
\frac{d}{d t}\left(\mathbf{u}^{\mathrm{b}}\right)^{\rho}=-\frac{1}{\triangle x}\left(\mathbf{g}^{\mathrm{b}}\left(\mathbf{u}^{\rho}(t)\right)-\mathbf{g}^{\mathrm{b}}\left(\mathbf{u}^{\rho-1}(t)\right)\right),
$$




$$
\frac{d}{d t}\left(\mathbf{u}^{\sharp}\right)^{\rho}=-\frac{1}{\triangle x}\left(\mathbf{g}^{\sharp}\left(\mathbf{u}^{\rho+1}(t)\right)-\mathbf{g}^{\sharp}\left(\mathbf{u}^{\rho}(t)\right)\right),
$$

$1 \leq \rho \leq M-1$, and also

$$
\begin{aligned}
\frac{d}{d t}\left(\mathbf{u}^{b}\right)^{M} & =-\frac{1}{\triangle x}\left(\mathbf{g}^{b}\left(\left(\mathbf{u}^{b}\right)^{M}(t)+\mathbf{e}^{\sharp}\right)-\mathbf{g}^{b}\left(\mathbf{u}^{M-1}(t)\right)\right), \\
\frac{d}{d t}\left(\mathbf{u}^{\sharp}\right)^{0} & =-\frac{1}{\triangle x}\left(\mathbf{g}^{\sharp}\left(\mathbf{u}^{1}(t)\right)-\mathbf{g}^{\sharp}\left(\mathbf{e}^{b}+\left(\mathbf{u}^{\sharp}\right)^{0}(t)\right)\right) .
\end{aligned}
$$

Equations (3.21), (3.22) define a system of ordinary differential equations in

$$
\mathbf{R}^{n(M-1)}
$$

while equations (3.23), (3.24) define two systems of ordinary differential equations in $\pi^{b}\left(\mathbf{R}^{n}\right)$ and $\pi^{\sharp}\left(\mathbf{R}^{n}\right)$, respectively. We remark that we have to interpret equation (3.21) for $\rho=1$ as

$$
\frac{d}{d t}\left(\mathbf{u}^{b}\right)^{1}=-\frac{1}{\triangle x}\left(\mathbf{g}^{b}\left(\mathbf{u}^{1}(t)\right)-\mathbf{e}^{b}\right),
$$

and equation (3.22) for $\rho=M-1$ as

$$
\frac{d}{d t}\left(\mathbf{u}^{\sharp}\right)^{M-1}=-\frac{1}{\triangle x}\left(\left(\mathbf{l}^{\sharp}-\mathbf{e}^{\sharp}\right)-\mathbf{g}^{\sharp}\left(\mathbf{u}^{M-1}(t)\right)\right),
$$

because of (3.18), (3.19) and the properties of $K$-fluxes (respectively, $\Delta^{i_{1}, i_{2}}$-fluxes). So, we have three initial value problems: the first formed by the system (3.21), (3.22) and the first equation in (3.20); the second given by (3.23) and the second equation in (3.20); the third constituted by (3.24) and the third equation in (3.20). The analysis of both the second and third problems is easier and follows by reasonings analogous to those in the analysis of the first. So, we will restrict ourselves to the study of the first initial value problem given by (3.21), (3.22) and (3.20). Set

$$
\Omega=\prod_{\rho=1}^{M-1} K \quad\left(\text { respectively, } \quad \prod_{\rho=1}^{M-1} \Delta^{i_{1}, i_{2}}\right)
$$

and let $X$ denote the vector field defined in $\Omega$ given by the right-hand side of (3.21), (3.22). It is easy to see that $X$ satisfies the hypotheses of Picard's theorem on the local existence and uniqueness of solutions to initial value problems for systems of ordinary differential equations. We will show that we can extend this local solution to a global one $\left(\mathbf{u}^{\rho}(t)\right)_{\rho=1}^{M-1}$ with $\mathbf{u}^{\rho}(t) \in K$ (respectively, $\Delta^{i_{1}, i_{2}}$ ), for all $t>0$, $\rho=1, \ldots, M-1$.

Let $\omega$ be a point in $\partial \Omega$, with $\omega=\left(\mathbf{u}^{\rho}\right)_{\rho=1}^{M-1}, \mathbf{u}^{\rho} \in K$ (respectively, $\left.\Delta^{i_{1}, i_{2}}\right)$. Then, there exists $\rho \in\{1, \ldots, M-1\}$ such that $\mathbf{u}^{\rho} \in \partial K$ (respectively, $\partial \Delta^{i_{1}, i_{2}}$ ). That is, for this $\rho$ we have one of the following three alternatives, the last one only in the case of $\Delta^{i_{1}, i_{2}}$-fluxes:

$$
\begin{aligned}
& u_{i}^{\rho}=0, \quad \text { for some } i \in\{1, \ldots, n\} \text { for some } i \in\{1, \ldots, n\} \\
& u_{i}^{\rho}=1, \\
& u_{i_{1}}^{\rho}+u_{i_{2}}^{\rho}=1 .
\end{aligned}
$$


We will have shown to be possible to extend indefinitely any local solution of the problem in question, with initial value in $K$ (respectively, $\Delta^{i_{1}, i_{2}}$ ), if we verify that

$$
\langle X(\omega), N(\omega)\rangle \leq 0
$$

for all $\omega \in \partial \Omega$, where $N(\omega)$ is the outward unit normal vector to $\partial \Omega$, defined in $\omega$. To show (3.28), let us assume for concreteness that in (3.25), (3.26) we have $i \in\left\{j_{1}, \ldots, j_{p}\right\}$, and in $(3.27)$ we have $\left\{i_{1}, i_{2}\right\} \subset\left\{j_{1}, \ldots, j_{p}\right\}$; the other cases follow similarly. Then, (3.28) follows because

$$
\langle X(\omega), N(\omega)\rangle=-X_{i}^{\rho}(\omega)=-\frac{1}{\triangle x} g_{i}\left(\mathbf{u}^{\rho-1}\right),
$$

if (3.25) holds,

$$
\langle X(\omega), N(\omega)\rangle=X_{i}^{\rho}(\omega)=-\frac{1}{\triangle x}\left(1-g_{i}\left(\mathbf{u}^{\rho-1}\right)\right),
$$

if (3.26) holds, and

$$
\langle X(\omega), N(\omega)\rangle=X_{i_{1}}^{\rho}(\omega)+X_{i_{2}}^{\rho}(\omega)=-\frac{1}{\triangle x}\left(1-g_{i_{1}}\left(\mathbf{u}^{\rho-1}\right)-g_{i_{2}}\left(\mathbf{u}^{\rho-1}\right)\right),
$$

if (3.27) holds, where $\omega=\left(\mathbf{u}^{\rho}\right)_{\rho=1}^{M-1}$, with $\mathbf{u}^{\rho} \in K$ (respectively, $\left.\Delta^{i_{1}, i_{2}}\right)$. So, we can extend the local solution to a global solution, $\left(\mathbf{u}^{\rho}(t)\right)_{\rho=1}^{M-1}$, of (3.21), (3.22), (3.20).

Again, we define, for $\varepsilon=\triangle x$,

$$
\mathbf{u}^{\varepsilon}(x, t)=\mathbf{u}^{\rho}(t), \quad \text { if }\left(\rho-\frac{1}{2}\right) \triangle x \leq x<\left(\rho+\frac{1}{2}\right) \triangle x, \quad 0 \leq \rho \leq M .
$$

By Tartar's theorem on the existence of Young measures we obtain a family of probability measures $\nu_{x, t}$, with $\operatorname{Supp} \nu_{x, t} \in K$ (respectively, $\left.\Delta^{i_{1}, i_{2}}\right)$, satisfying (2.10) for all $h \in C(K)$ (respectively, $C\left(\Delta^{i_{1}, i_{2}}\right)$ ). We prove that $\nu_{x, t}$ is a m-v solution to (1.5)-(1.8) exactly as we did for the Young measure obtained from the solutions of the scheme (3.2)-(3.6).

\section{Dynamics: Weak convergence of the time-aVerages}

Here we study the asymptotic behavior of the m-v solutions of (1.5)-(1.8). As before, let $\mathbf{f} \in \mathcal{F}^{+}(K)$ (respectively, $\mathcal{F}^{+}\left(\Delta^{i_{1}, i_{2}}\right)$ ) be related with $\mathbf{g}$ by $\mathbf{g}=S \mathbf{f}$, for some $S \in \mathcal{S}(K)$. By Lemma 1.5, if $\mathbf{f} \in \mathcal{F}^{+}\left(\Delta^{i_{1}, i_{2}}\right)$ we can extend it to $K$ as an element of $\mathcal{F}^{+}(K)$, so we will always consider $\mathbf{f} \in \mathcal{F}^{+}(K)$; this will be sufficient for the discussion in this section.

Let us define

$$
\bar{f}_{i}(s)=f_{i}\left(\mathbf{e}+\left(s-\delta_{i}\right) \mathbf{e}_{i}\right), \quad s \in[0,1],
$$

where $\mathbf{e}=\mathbf{e}^{\mathrm{b}}+\mathbf{e}^{\sharp}=\left(\delta_{1}, \ldots, \delta_{n}\right), \delta_{i}=0$ or 1 , is a vertex of coerciveness of $\mathbf{f}$. The $\bar{f}_{i}$ satisfy: $\bar{f}_{i}\left(\left[0,1-\varepsilon_{0}\right)\right) \cap \bar{f}_{i}\left(\left[1-\varepsilon_{0}, 1\right]\right)=\emptyset$ and $\bar{f}_{i} \mid\left[1-\varepsilon_{0}, 1\right]$ is strictly increasing, with $\bar{f}_{i}(1)=1$.

In this section we will be interested in proving the following result about the dynamics of the m-v solutions of (1.5)-(1.8). 
4.1 Theorem. Let $\nu_{x, t}$ be a m-v solution of (1.5)-(1.8) and set

$$
\left\langle\mu_{T}^{x}, .\right\rangle=\frac{1}{T} \int_{0}^{T}\left\langle\nu_{x, t}, .\right\rangle d t .
$$

Then, for a.e. $x \in[0, L], \mu_{T}^{x} \rightarrow \delta_{\mathbf{e}}$, as $T \rightarrow \infty$, where $\delta_{\mathbf{e}}$ is the Dirac measure concentrated at $\mathbf{e}=\mathbf{e}^{\mathrm{b}}+\mathbf{e}^{\sharp}$. Further, the rates of convergence of the limits

$$
\lim _{T \rightarrow \infty}\left\langle\mu_{T}^{x}, \bar{f}_{i}\right\rangle=\delta_{i}
$$

can be estimated by the order of the envelope of a family of functions $\rho^{\varepsilon}(T)$ of the form

$$
\rho^{\varepsilon}(T)=\varepsilon+C^{i}(\varepsilon) T^{-1},
$$

where $C^{i}(\varepsilon)$ is a function satisfying $C^{i}(\varepsilon) \rightarrow \infty$ as $\varepsilon \rightarrow 0$ and depends only on the map $\mathbf{f}$, for each $i=1, \ldots, n$. More yet, the rates of convergence of the limits

$$
\lim _{T \rightarrow \infty}\left\langle\mu_{T}^{x}, u_{i}\right\rangle=\delta_{i}
$$

for a.e. $x \in[0,1], i=1, \ldots, n$, can always be estimated using properties of the function $\bar{f}_{i}$ and estimates for the rates of convergence of the limits (4.2).

Theorem 4.1 will follow from Proposition 1.7, more precisely, from (1.4), and some lemmas about weak convergence of probability measures to Dirac measures that we now state and prove.

4.2 Lemma. Consider a one-parameter family of probability Borel measures defined on the interval $[0,1], \mu_{T} \in \mathbf{P}([0,1]), 0<T<\infty$. Let $\sigma:[0,1] \rightarrow[0,1]$ be a Borel function satisfying: for some $\varepsilon_{0}>0, \sigma\left(\left[0,1-\varepsilon_{0}\right)\right) \cap \sigma\left(\left[1-\varepsilon_{0}, 1\right]\right)=\emptyset$ and $\sigma \mid\left[1-\varepsilon_{0}, 1\right]$ is injective increasing, with $\sigma(1)=1$. Assume

$$
\lim _{T \rightarrow \infty}\left\langle\mu_{T}, \sigma\right\rangle=1 \text {. }
$$

Then $\mu_{T} \rightarrow \delta_{\{1\}}$, where $\delta_{\{1\}}$ is the Dirac measure on $[0,1]$, concentrated at 1 . Suppose, further, that (4.5) converges at a rate $O\left(T^{-\alpha}\right), \alpha>0$. Then, for the rate of convergence of the limit

$$
\lim _{T \rightarrow \infty}\left\langle\mu_{T}, i d\right\rangle=1,
$$

where id denotes the identity map on $[0,1], s \mapsto s$, we have the following:

1. The rate of convergence of (4.6) can always be estimated by the order of the envelope of a family of functions $\rho^{\varepsilon}(T)$ of the form

$$
\rho^{\varepsilon}(T)=\varepsilon+\frac{C}{1-\sigma(1-\varepsilon)} T^{-\alpha},
$$

for some $C>0$.

2. If we can choose $\varepsilon_{0}$ sufficiently small so that $\sigma \mid\left[1-\varepsilon_{0}, 1\right]$ is continuously differentiable and $\sigma^{\prime}>0$ in $\left[1-\varepsilon_{0}, 1\right]$, then (4.6) is realized with, at least, the same rate of convergence $O\left(T^{-\alpha}\right)$ as (4.5).

3. If $\rho:[0,1] \rightarrow[0,1]$ is convex with $\rho(0)=0$ and $\rho \circ \sigma(s) \leq s, s \in[0,1]$, then (4.6) is attained at a rate at least $O\left(1-\rho\left(1-O\left(T^{-\alpha}\right)\right)\right)$.

Before proving Lemma 4.2 we will state the analogous result for probability measures on $[0,1]$ converging weakly to Dirac measures concentrated at 0 , which follows immediately from Lemma 4.2. 
4.3 Lemma. Consider a one-parameter family of probability Borel measures defined on the interval $[0,1], \mu_{T} \in \mathbf{P}([0,1]), 0<T<\infty$. Let $\tilde{\sigma}:[0,1] \rightarrow[0,1]$ be a Borel function satisfying: for some $\varepsilon_{0}>0, \tilde{\sigma}\left(\left[0, \varepsilon_{0}\right]\right) \cap \tilde{\sigma}\left(\left(\varepsilon_{0}, 1\right]\right)=\emptyset$ and $\tilde{\sigma} \mid\left[0, \varepsilon_{0}\right]$ is injective increasing, with $\tilde{\sigma}(0)=0$. Assume

$$
\lim _{T \rightarrow \infty}\left\langle\mu_{T}, \tilde{\sigma}\right\rangle=0 .
$$

Then $\mu_{T} \rightarrow \delta_{\{0\}}$. Further, if (4.7) converges with a rate $O\left(T^{-\alpha}\right), \alpha>0$, then, with respect to the rate of convergence of

$$
\lim _{T \rightarrow \infty}\left\langle\mu_{T}, t\right\rangle=0,
$$

where $t$ denotes the identity map $t \mapsto t$ on $[0,1]$, we have the following:

1. The rate of convergence of (4.8) can always be estimated by the order of the envelope of a family of functions of the form

$$
\rho^{\varepsilon}(T)=\varepsilon+\frac{C}{\tilde{\sigma}(\varepsilon)} T^{-\alpha},
$$

for some $C>0$.

2. If we can choose $\varepsilon_{0}$ sufficiently small so that $\tilde{\sigma} \mid\left[0, \varepsilon_{0}\right]$ is continuously differentiable and $\tilde{\sigma}^{\prime}>0$ in $\left[0, \varepsilon_{0}\right]$, then (4.8) is achieved with, at least, the same rate of convergence $O\left(T^{-\alpha}\right)$ which estimates the rate of (4.7).

3. If $\tilde{\rho}:[0,1] \rightarrow[0,1]$ is a concave function with $\tilde{\rho}(1)=1$ and $\tilde{\rho} \circ \tilde{\sigma}(t) \geq t$, $t \in\left[0, \varepsilon_{0}\right]$, then (4.8) is realized at a rate at least $O\left(\tilde{\rho}\left(O\left(T^{-\alpha}\right)\right)\right)$.

We easily see that Lemma 4.3 follows from Lemma 4.2 by making $s=1-t$ (with a corresponding pull-back of the measures $\left.\mu_{T}\right)$ and defining $\sigma(s)=1-\tilde{\sigma}(t)$.

Proof of Lemma 4.2. Let $\varepsilon_{0}^{\prime}$ be such that $\sigma\left(\left[1-\varepsilon_{0}, 1\right]\right)=\left[1-\varepsilon_{0}^{\prime}, 1\right]$. We first note that since $\sigma\left(\left[0,1-\varepsilon_{0}\right)\right) \cap \sigma\left(\left[1-\varepsilon_{0}, 1\right]\right)=\emptyset$ then we must have

$$
\chi_{\left[1-\varepsilon_{0}^{\prime}, 1\right]} \circ \sigma=\chi_{\left[1-\varepsilon_{0}, 1\right]}:=\chi^{+},
$$

and

$$
\chi_{\left[0,1-\varepsilon_{0}^{\prime}\right)} \circ \sigma=\chi_{\left[0,1-\varepsilon_{0}\right)}:=\chi^{-} .
$$

Consider

$$
\begin{gathered}
\theta_{1}=\liminf _{T \rightarrow \infty}\left\langle\mu_{T}, \chi^{+}\right\rangle, \\
\theta_{2}=\limsup _{T \rightarrow \infty}\left\langle\mu_{T}, \chi^{-}\right\rangle .
\end{gathered}
$$

We first prove that $\theta_{1}=1$ and $\theta_{2}=0$, and so, in (4.9) and (4.10), we can take limits instead of liminf and limsup, respectively. From

$$
\left\langle\mu_{T}, \chi^{+}\right\rangle+\left\langle\mu_{T}, \chi^{-}\right\rangle=1 \text {, }
$$


we immediately get $\theta_{1}+\theta_{2}=1$. We also have

$$
\begin{aligned}
\left\langle\mu_{T}, \sigma\right\rangle & =\left\langle\mu_{T}, \chi^{-} \cdot \sigma\right\rangle+\left\langle\mu_{T}, \chi^{+} \cdot \sigma\right\rangle \\
& \leq\left(1-\varepsilon_{0}^{\prime}\right)\left\langle\mu_{T}, \chi^{-}\right\rangle+\left\langle\mu_{T}, \chi^{+}\right\rangle .
\end{aligned}
$$

So, given $\varepsilon>0$ sufficiently small, for $T>0$ sufficiently large we have

$$
\left\langle\mu_{T}, \sigma\right\rangle \leq\left(1-\varepsilon_{0}^{\prime}\right)\left(\theta_{2}+\varepsilon\right)+\left\langle\mu_{T}, \chi^{+}\right\rangle .
$$

Taking liminf on both sides of this inequality and making $\varepsilon \rightarrow 0$ we obtain

$$
1 \leq\left(1-\varepsilon_{0}^{\prime}\right) \theta_{2}+\theta_{1},
$$

and this is possible only if $\theta_{2}=0$ and $\theta_{1}=1$. So, we have

$$
\lim _{T \rightarrow \infty}\left\langle\mu_{T}, \chi^{+}\right\rangle=1
$$

and

$$
\lim _{T \rightarrow \infty}\left\langle\mu_{T}, \chi^{-}\right\rangle=0 .
$$

Now, it is clear that, by the same reasonings adopted above, we have, for any $0<\varepsilon \leq \varepsilon_{0}$,

$$
\lim _{T \rightarrow \infty}\left\langle\mu_{T}, \chi_{[1-\varepsilon, 1]}\right\rangle=1 .
$$

Then, Tchebychev's inequality gives us

$$
\left\langle\mu_{T}, \chi_{[1-\varepsilon, 1]}\right\rangle \leq \frac{1}{1-\varepsilon}\left\langle\mu_{T}, s\right\rangle .
$$

Taking liminf on both sides of (4.16), we get

$$
1-\varepsilon \leq \liminf _{T \rightarrow \infty}\left\langle\mu_{T}, s\right\rangle .
$$

Since $\varepsilon \leq \varepsilon_{0}$ can be made arbitrarily small, we obtain

$$
1 \leq \liminf _{T \rightarrow \infty}\left\langle\mu_{T}, s\right\rangle
$$

and this implies (4.6). Now, given any positive integer $n$, if $h(s)=s^{n}$, then by the Mean Value Theorem we have

$$
0 \leq\left\langle\mu_{T}, 1-h\right\rangle \leq C\langle\mu, 1-s\rangle,
$$

for $C>0$ independent of $T$, and so

$$
\lim _{T \rightarrow \infty}\left\langle\mu_{T}, h\right\rangle=1=\left\langle\delta_{\{1\}}, h\right\rangle .
$$


Hence, by linearity and density (Weierstrass theorem) we arrive at $\mu_{T} \rightarrow \delta_{\{1\}}$, concluding the proof of the first part of Lemma 4.2.

We now pass to the proof of the assertions (1), (2) and (3) in the statement of Lemma 4.2. First, by (4.11) and (4.12) we see that

$$
\left\langle\mu_{T}, \sigma\right\rangle \leq 1-\varepsilon_{0}^{\prime}\left\langle\mu_{T}, \chi^{-}\right\rangle,
$$

and, since $\left\langle\mu_{T}, \sigma\right\rangle=1-O\left(T^{-\alpha}\right)$, we have that $\left\langle\mu_{T}, \chi^{-}\right\rangle$is, at most, $O\left(T^{-\alpha}\right)$. In fact, by (4.17) we see that we can find a constant $C>0$ such that

$$
0 \leq\left\langle\mu_{T}, \chi^{-}\right\rangle \leq \frac{C}{\varepsilon_{0}^{\prime}} T^{-\alpha} .
$$

So, by (4.11), we have

$$
1 \geq\left\langle\mu_{T}, \chi^{+}\right\rangle \geq 1-\frac{C}{\varepsilon_{0}^{\prime}} T^{-\alpha},
$$

for some $C>0$. Now, (4.19) and (4.16) imply that, for some $C>0$, we have

$$
1 \geq\left\langle\mu_{T}, s\right\rangle \geq 1-\varepsilon-\frac{C}{1-\sigma(1-\varepsilon)} T^{-\alpha},
$$

and this proves assertion (1).

We pass to assertion (2). Observe first that

$$
\lim _{T \rightarrow \infty}\left\langle\mu_{T}, \chi^{+} \sigma\right\rangle=1,
$$

and this limit is realized at least at a rate $O\left(T^{-\alpha}\right)$. Indeed, we have

$$
0 \leq\left\langle\mu_{T}, \chi^{-} \sigma\right\rangle \leq\left\langle\mu_{T}, \chi^{-}\right\rangle
$$

and so

$$
\lim _{T \rightarrow \infty}\left\langle\mu_{T}, \chi^{-} \sigma\right\rangle=0,
$$

with a rate of convergence at least $O\left(T^{-\alpha}\right)$. Hence, by (4.11), we have (4.20) and the asserted estimate for the rate of convergence.

For obtaining assertion (2) we observe that, by the Mean Value Theorem, we have

$$
\left\langle\mu_{T}, \chi^{+}(1-\sigma)\right\rangle \geq C\left\langle\mu_{T}, 1-s\right\rangle \geq 0,
$$

for some $C>0$ independent of $T$, and, so, follows the assertion.

For the assertion (3), we note that, by Jensen's inequality, we have

$$
\begin{aligned}
\rho\left(\left\langle\mu_{T}, \chi^{+} \sigma\right\rangle\right) & \leq\left\langle\mu_{T}, \chi^{+} \rho \circ \sigma\right\rangle \\
& \leq\left\langle\mu_{T}, s\right\rangle \leq 1 .
\end{aligned}
$$

Hence, assertion (3) follows. The lemma is then proved. 
Remark. In general, assertions (2) and (3) in Lemma 4.2 give better estimates for the rate of convergence of (4.6) than those obtained by assertion (1). This can be seen taking as example the case where $\alpha=1$ and $\sigma(s)=s$. In this case

$$
\rho^{\varepsilon}(T)=\varepsilon+\frac{C}{\varepsilon} T^{-1},
$$

and the envelope is obtained from the equation

$$
1-\frac{C}{\varepsilon^{2}} T^{-1}=-\frac{C}{\varepsilon} T^{-2},
$$

which gives $\varepsilon=O\left(T^{-\frac{1}{2}}\right)$, and, so, the envelope is $O\left(T^{-\frac{1}{2}}\right)$. This is far from the correct rate which, by assumption, was $O\left(T^{-1}\right)$.

The two lemmas above and the techniques used in their proofs can be applied to obtain results in more than one dimension. The following result shows this and together with (1.14) in Proposition 1.7 gives the proof of Theorem 4.1.

4.4 Proposition. Let $K=\prod_{i=1}^{n}[0,1]$ and $\mu_{T} \in \mathbf{P}(K), 0<T<\infty$. Suppose $\mathbf{f} \in \mathcal{F}^{+}(K)$ with $\mathbf{e}$ a vertex of coerciveness, and assume that

$$
\lim _{T \rightarrow \infty}\left\langle\mu_{T}, \mathbf{f}\right\rangle=\mathbf{e} .
$$

Then, $\mu_{T} \rightarrow \delta_{\mathbf{e}}$. Further, if the limit (4.21) is attained at a rate of convergence $O\left(T^{-\alpha}\right)$, for some $0<\alpha \leq 1$, then we can obtain estimates for the rates of convergence of the limits

$$
\lim _{T \rightarrow \infty}\left\langle\mu_{T}, \bar{f}_{i}\right\rangle=\delta_{i}, \quad i=1, \ldots, n,
$$

by the order of the envelope of a family of functions $\rho^{\varepsilon}(T)$, of the form

$$
\rho^{\varepsilon}(T)=\varepsilon+C(\varepsilon) T^{-\alpha},
$$

where $C(\varepsilon)$ is a function such that $C(\varepsilon) \rightarrow \infty$ as $\varepsilon \rightarrow 0$, which depends only on the map $\mathbf{f}$. In particular, we can obtain estimates for the rates of convergence of the limits

$$
\lim _{T \rightarrow \infty}\left\langle\mu_{T}, u_{i}\right\rangle=\delta_{i}, \quad i=1, \ldots, n,
$$

by using assertions (1), (2), (3) of Lemma 4.2, with $\sigma=\bar{f}_{i}$, if $\delta_{i}=1$, or the corresponding assertions in Lemma 4.3, with $\tilde{\sigma}=\bar{f}_{i}$, if $\delta_{i}=0$.

Remark. The order of the envelope of a family of functions like (4.23) is often easy to obtain. For instance, if $C(\varepsilon)=\frac{C}{\varepsilon^{\gamma}}, \gamma>1$, it is easy to see that it is $O\left(T^{-\frac{\alpha}{\gamma+1}}\right)$.

Proof. Without loss of generality, we can take $\mathbf{e}=(1,1, \ldots, 1)$, as will become clear by the proof. We make another simplification assuming $\mathbf{f}$ to be a homeomorphism on $K$. Again, it will be evident from the proof that this does not imply any loss of generality. So, let $\varepsilon^{\prime}>0$ be given. We denote

$$
\begin{aligned}
& \chi_{i}^{-} \stackrel{\text { def }}{=} \chi_{\left[0,1-\varepsilon^{\prime}\right)} \circ f_{i}, \\
& \chi_{i}^{+} \stackrel{\text { def }}{=} \chi_{\left[1-\varepsilon^{\prime}, 1\right]} \circ f_{i}, \quad i=1, \ldots, n .
\end{aligned}
$$


Proceeding as in the proof of (4.13), (4.14), we prove that

$$
\begin{aligned}
& \lim _{T \rightarrow \infty}\left\langle\mu_{T}, \chi_{i}^{-}\right\rangle=0, \\
& \lim _{T \rightarrow \infty}\left\langle\mu_{T}, \chi_{i}^{+}\right\rangle=1,
\end{aligned}
$$

with a rate at least equal to that of $(4.21), i=1, \ldots, n$. Now, given any $i \in$ $\{1, \ldots, n\}$ we have

$$
\lim _{T \rightarrow \infty}\left\langle\mu_{T},\left(\prod_{\substack{j=1 \\ j \neq i}}^{n} \chi_{j}^{+}\right) f_{i}\right\rangle=1 .
$$

Indeed, given $j \in\{1, \ldots, n\}$, we have

$$
0 \leq\left\langle\mu_{T}, \chi_{j}^{-} \cdot f_{i}\right\rangle \leq\left\langle\mu_{T}, \chi_{j}^{-}\right\rangle .
$$

Since the right side of the last inequality goes to zero when $T \rightarrow \infty$ we get

$$
\lim _{T \rightarrow \infty}\left\langle\mu_{T}, \chi_{j}^{-} \cdot f_{i}\right\rangle=0,
$$

and so, by (4.21), we get

$$
\lim _{T \rightarrow \infty}\left\langle\mu_{T}, \chi_{j}^{+} f_{i}\right\rangle=1 .
$$

Similarly, if we also take $k \in\{1, \ldots, n\}$ we obtain

$$
\lim _{T \rightarrow \infty}\left\langle\mu_{T}, \chi_{k}^{+} \chi_{j}^{+} f_{i}\right\rangle=1 .
$$

Repeating this procedure as many times as we need we arrive at (4.27). Note that if the rate of convergence of (4.21) is $O\left(T^{-\alpha}\right)$, then the rate of (4.27) can also be estimated by $O\left(T^{-\alpha}\right)$.

In (4.22), (4.24) and in what follows, $\mu_{T}$ applied to a function of only one variable means that we have to consider this function as one of the $n$ variables which depends only on one of the $n$ variables (with this we avoid speaking explicitly of projections of measures). So, we have

$$
\begin{aligned}
\left\langle\mu_{T}, \bar{f}_{i}\right\rangle=\left\langle\mu_{T},\left(\prod_{\substack{j=1 \\
j \neq i}}^{n} \chi_{j}^{+}\right) f_{i}\right\rangle \\
+\left\langle\mu_{T},\left(\prod_{\substack{j=1 \\
j \neq i}}^{n} \chi_{j}^{+}\left(\bar{f}_{i}-f_{i}\right)\right\rangle\right. \\
+\left\langle\mu_{T},\left(1-\prod_{\substack{j=1 \\
j \neq i}}^{n} \chi_{j}^{+}\right) \bar{f}_{i}\right\rangle .
\end{aligned}
$$


Now, given $\varepsilon>0$, for $\varepsilon^{\prime}$ sufficiently small we can make the second term of the sum in the right-hand side of (4.28) less than $\varepsilon$ in absolute value. For the third term, we have

$$
0 \leq\left\langle\mu_{T},\left(1-\prod_{\substack{j=1 \\ j \neq i}}^{n} \chi_{j}^{+}\right) \bar{f}_{i}\right\rangle \leq\left\langle\mu_{T}, 1-\prod_{\substack{j=1 \\ j \neq i}}^{n} \chi_{j}^{+}\right\rangle,
$$

and the term on the right of the last inequality above goes to zero, as it is immediate from (4.26). Then, (4.28) gives

$$
\liminf _{T \rightarrow \infty}\left\langle\mu_{T}, \bar{f}_{i}\right\rangle \geq 1-\varepsilon .
$$

So, making $\varepsilon \rightarrow 0$, we get

$$
\lim _{T \rightarrow \infty}\left\langle\mu_{T}, \bar{f}_{i}\right\rangle=1, \quad i=1, \ldots, n .
$$

If the limit in (4.21) is attained with a rate of convergence $O\left(T^{-\alpha}\right)$, it follows from (4.28) that we can find a function of $\varepsilon, C(\varepsilon)$, depending only on the map $\mathbf{f}$, so that

$$
\left\langle\mu_{T}, \bar{f}_{i}\right\rangle \geq 1-\varepsilon-C(\varepsilon) T^{-\alpha} .
$$

Then, finding the envelope of the family $\rho^{\varepsilon}(T)=\varepsilon+C(\varepsilon) T^{-\alpha}$, we obtain an estimate for the rate of convergence of (4.22) as asserted.

To conclude the proof, we apply Lemma 4.2 with $\bar{f}_{i}$ as $\sigma$ to get

$$
\lim _{T \rightarrow \infty}\left\langle\mu_{T}, u_{i}\right\rangle=1, \quad i=1, \ldots, n .
$$

To get that $\mu_{T} \rightarrow \delta_{\mathbf{e}}$ we note that if $h\left(u_{1}, \ldots, u_{n}\right)=u_{1}^{k_{1}} \ldots u_{n}^{k_{n}}$, then there exists $C>0$ such that

$$
0 \leq 1-h \leq C \sum_{i=1}^{n}\left(1-u_{i}\right),
$$

by the Mean Value Theorem and so

$$
0 \leq\left\langle\mu_{T}, 1-h\right\rangle \leq C \sum_{i=1}^{n}\left\langle\mu_{T}, 1-u_{i}\right\rangle,
$$

which implies

$$
\lim _{T \rightarrow \infty}\left\langle\mu_{T}, h\right\rangle=1=\left\langle\delta_{\mathbf{e}} \cdot h\right\rangle .
$$

Then, by linearity and density (Weierstrass Theorem) we arrive at the desired result.

Proof of Theorem 4.1. By (1.14) in Proposition 1.6 we have, for a.e. $x \in[0, L]$,

$$
\lim _{T \rightarrow \infty}\left\langle\mu_{T}^{x}, \mathbf{f}\right\rangle=\mathbf{e},
$$

with a rate of convergence $O\left(T^{-1}\right)$. So, we can apply Proposition 4.4 to get all the assertions in the statement of Theorem 4.1. 
Remarks. 1. The estimates for the rates of the expected values of the dependent variables can usually be obtained by combining the use of peculiarities of the map $\mathbf{f}$ with the obtainment of the order of envelopes of families of functions. For instance, consider the map $\mathbf{f}=\left(f_{1}, f_{2}\right)$ given by

$$
f_{1}(u, v)=\frac{u^{2}}{u^{2}+v^{2}+(1-u-v)^{2}}, \quad f_{2}(u, v)=\frac{v^{2}}{u^{2}+v^{2}+(1-u-v)^{2}},
$$

which appears in a simple model for three-phase flows in porous media (cf. [5]). Assume that for a family $\mu_{T} \in \mathbf{P}(\Delta), 0<T<\infty$, where $\Delta=\{(u, v): 0 \leq u \leq$ $1,0 \leq v \leq 1,0 \leq u+v \leq 1\}$, we have

$$
\lim _{T \rightarrow \infty}\left\langle\mu_{T}, \mathbf{f}\right\rangle=(1,0)
$$

with a rate of convergence $O\left(T^{-1}\right)$, as it occurs if the $\mu_{T}$ are the time-averages of a measure-valued solution to the initial boundary value problem modelling the flow of water, gas and oil in a reservoir submitted to the constant injection of pure water (see [3]).

Then, since $C_{1} v^{2} \leq f_{2}(u, v) \leq C_{2} v^{2}$, for some constants $C_{1}, C_{2}>0$, we easily see that

$$
\lim _{T \rightarrow \infty}\left\langle\mu_{T}, v^{2}\right\rangle=0
$$

with a rate $O\left(T^{-1}\right)$. By Jensen's inequality we have

$$
\left\langle\mu_{T}, v^{2}\right\rangle^{\frac{1}{2}} \geq\left\langle\mu_{T}, v\right\rangle \geq 0
$$

which gives

$$
\lim _{T \rightarrow \infty}\left\langle\mu_{T}, v\right\rangle=0
$$

at a rate at least $O\left(T^{-\frac{1}{2}}\right)$.

Now, by Tchebychev's inequality, we have, for $\varepsilon>0$ small,

$$
0 \leq\left\langle\mu_{T}, \chi_{(\varepsilon, 1]}(v)\right\rangle \leq \frac{1}{\varepsilon^{2}}\left\langle\mu_{T}, v^{2}\right\rangle .
$$

Hence,

$$
\lim _{T \rightarrow \infty}\left\langle\mu_{T}, \chi_{(\varepsilon, 1]}(v)\right\rangle=0
$$

at a rate $O\left(T^{-1}\right)$, and so

$$
\lim _{T \rightarrow \infty}\left\langle\mu_{T}, \chi_{[0, \varepsilon]}(v)\right\rangle=1,
$$

with the same rate. We, then, get

$$
\lim _{T \rightarrow \infty}\left\langle\mu_{T}, \chi_{[0, \varepsilon]}(v) f_{1}\right\rangle=1,
$$

at a rate $O\left(T^{-1}\right)$. So, we obtain

$$
\begin{aligned}
\left\langle\mu_{T}, \bar{f}_{1}\right\rangle & \geq\left\langle\mu_{T}, \chi_{[0, \varepsilon]}(v) \bar{f}_{1}\right\rangle \\
& \geq\left\langle\mu_{T}, \chi_{[0, \varepsilon]}(v) f_{1}\right\rangle-C_{2} \varepsilon \\
& \geq 1-\frac{C_{1}}{\varepsilon^{2}} T^{-1}-C_{2} \varepsilon .
\end{aligned}
$$


Thus, we arrive at

$$
\lim _{T \rightarrow \infty}\left\langle\mu_{T}, \bar{f}_{1}\right\rangle=1
$$

the rate of convergence being estimated by the order of the envelope of

$$
\rho^{\varepsilon}(T)=\frac{C_{1}}{\varepsilon^{2}} T^{-1}+C_{2} \varepsilon,
$$

which is easily seen to be $O\left(T^{-\frac{1}{3}}\right)$. Then, the rate of convergence of

$$
\lim _{T \rightarrow \infty}\left\langle\mu_{T}, u\right\rangle=1
$$

can be estimated by $O\left(1-\bar{f}_{1}^{-1}\left(1-O\left(T^{-\frac{1}{3}}\right)\right)\right)$, which is the same as $O\left(T^{-\frac{1}{6}}\right)$, since $\bar{f}_{1}^{\prime}(1)=0$ and $\bar{f}_{1}^{\prime \prime}(1)<0$.

2. By Lemma 3.1 we see that the asymptotic behavior of the expected values of $\mathrm{m}-\mathrm{v}$ solutions to the problem (1.5)-(1.8) is already presented by the approximate solutions given by the finite difference schemes defined in section 3 . This fact allows the investigation of sharp estimates for the rates of convergence on the basis of the issues of numerical experiments.

\section{ACKNOWLEDGEMENT}

The author wishes to thank Jorge Araújo, from UFRGS, for the discussions and valuable sugestions during the preparation of this work.

\section{REFERENCES}

1. K.N. Chueh, C.C. Conley, J.A. Smoller, Positively invariant regions for systems of nonlinear diffusion equations, Indiana University Mathematics Journal 26 (1977), no. 2, 372-411. MR 55:3541

2. R.J. DiPerna, Measure-valued solutions to conservation laws, Arch. Rat. Mech. Anal. 8 (1985), 223-270. MR 86g:35121

3. H. Frid, Existence and asymptotic behavior of measure-valued solutions for three-phase flows in porous media, J. Math. Anal. Appl., (to appear).

4. D. Hoff, J.A. Smoller, Solutions in the large for certain nonlinear parabolic systems, Ann. Inst. Henri Poincaré-Analyse non linéaire 2 (1985), no. 3, 213-235. MR 87b:35078

5. E. Isaacson, D. Marchesin, B.V. Plohr, J.B. Temple, Multiphase flow models with singular Riemann problems, Computational and Applied Mathematics (1991). MR 94h:35212

6. S.N. Kruskov, First-order quasilinear equations with several space variables, Mat. Sb. 123 (1970), 228-255, English translation:; Math. USSR Sb. 10 (1970), 217-273. MR 42:2159

7. P.D. Lax, B. Wendroff, Systems of conservation laws, Comm. Pure Appl. Math. XIII (1960), 217-237. MR 22:11523

8. D.W. Peaceman, Fundamentals of Numerical Reservoir Simulation, Elsevier, AmsterdamNew York, 1977.

9. S. Saks, Theory of the Integral, Warsaw, 1937.

10. J.A. Smoller, Shock Waves and Reaction-Diffusion Equations, Springer-Verlag, New York, 1983. MR 84d:35002

11. L. Tartar, Compensated compactness and applications to partial differential equations, Research Notes in Mathematics, Nonlinear Analysis and Mechanics: Heriot-Watt Symposium, ed. R.J. Knops, vol. 4, Pitman Press, New York, 1979, pp. 136-212. MR 81m:35014

Instituto de Matemática, Universidade Federal do Rio de Janeiro, C. Postal 68530, CEP 21945, Rio de Janeiro, Brazil 